\title{
COMBINING DEFLATION AND NESTED ITERATION FOR COMPUTING MULTIPLE SOLUTIONS OF NONLINEAR VARIATIONAL PROBLEMS*
}

\author{
J. H. ADLER ${ }^{\dagger}$, D. B. EMERSON ${ }^{\dagger}$, P. E. FARRELL ${ }^{\ddagger}$, AND S. P. MACLACHLAN $\S$
}

\begin{abstract}
Many physical systems support multiple equilibrium states that enable their use in modern science and engineering applications. Having the ability to reliably compute such states facilitates more accurate physical analysis and understanding of experimental behavior. This paper adapts and extends a deflation technique for the computation of multiple distinct solutions in the context of nonlinear systems and applies the method to the modeling of equilibrium configurations of nematic and cholesteric liquid crystals. In particular, the deflation approach is interwoven with nested iteration, creating an efficient and effective method that further enables the discovery of distinct solutions. The combined methodology is applied as part of an overall free-energy variational approach within the framework of optimization of a functional with constraints imposed via Lagrange multipliers. A key feature in the combined algorithm is the reuse of effective preconditioners designed for the undeflated systems within the Newton iteration for the deflated systems. Four numerical experiments are performed, demonstrating the efficacy and accuracy of the algorithm in detecting important physical phenomena, including bifurcation and disclination behaviors.
\end{abstract}

Key words. liquid crystal simulation, deflation methods, energy optimization, nested iteration, distinct solutions

AMS subject classifications. 76A15, 65N30, 49M15, 65N22, 65N55

1. Introduction. It is well known that many systems of nonlinear partial differential equations (PDEs) permit multiple solutions. The numerical solution of such systems is of key interest in many settings, particularly for bistable and multistable physical systems, where understanding the solution set is crucial to the design and optimization of physical devices. In [22], a deflation methodology was proposed to enable the sequential discovery of distinct solutions to nonlinear differential equations, based on modifying the typical Newton iteration applied to their discretizations. The resulting iteration is demonstrated therein to be efficient, with roughly the same amount of computational effort required to find each additional solution, and to successfully discover numerous solutions to classical problems, such as an Allen-Cahn equation and the Navier-Stokes equations. In this paper, we adapt and expand the deflation methodology by combining it with nested iteration (NI) $[4,6,12,37$, 44], a powerful approach to reducing computational cost in the solution of nonlinear PDEs. To demonstrate the performance of the resulting algorithm, we apply it to models in liquid crystal theory, as an example of the coupled systems that arise in multiphysics simulation.

In general, the deflation methodology sequentially modifies the nonlinear problem to be solved by eliminating previously known solutions from consideration. This allows for successive discovery of distinct solutions to the nonlinear system. In practice,

\footnotetext{
*Revised November 26, 2016

$\dagger$ Department of Mathematics, Tufts University, Medford, MA 02155 (james.adler@tufts.edu, david.emerson@tufts.edu).

${ }^{\ddagger}$ Mathematical Institute, University of Oxford, Oxford OX2 6GG, United Kingdom (patrick.farrell@maths.ox.ac.uk). This author's work was funded by EPSRC grants EP/K030930/1, EP/M011151/1, and a Center of Excellence grant from the Research Council of Norway to the Center for Biomedical Computing at Simula Research Laboratory.

$\S$ Department of Mathematics and Statistics, Memorial University of Newfoundland, St. John's, Newfoundland and Labrador A1C 5S7, Canada (smaclachlan@mun.ca). This author's work was partially supported by an NSERC Discovery Grant.
} 
the deflation approach is highly effective in locating several distinct solutions. Here, we examine the method's performance in the context of constrained variational formulations arising in free-energy optimization. Such a framework is central to simulating various multiphysics applications such as liquid crystals (addressed here) and others, including ferromagnetics [32] and magnetohydrodynamics [29]. In the context of these types of problems, multiple local extrema and saddle-point solutions satisfying the given constraints may be present. Locating distinct solutions thus both reveals configurations with physical relevance, such as defect arrangements in the case of liquid crystals considered here, and facilitates computing global extrema with a higher level of confidence than otherwise possible.

The success of the deflation methodology relies on the efficiency of the underlying nonlinear and linear solvers. For the linear solvers, the deflation methodology is naturally attractive, as it allows for the preservation of the sparsity patterns seen in typical finite-element simulations as well as the use of existing fast solvers for the linearized systems. To address efficiency of the underlying nonlinear solvers, in this paper, we extend the deflation technique by integrating it with an NI approach, as is commonly used to improve that efficiency. NI is an important tool for the efficient numerical solution of nonlinear PDEs [44]. The system is first solved on a coarse level, where computation is cheap. A series of refinement steps are then taken, interpolating the coarse-grid solution to a finer mesh and using this as an initial guess for the fine-grid problem. A key advantage is that these interpolated approximations are typically very good initial guesses for Newton's method on the finer grids, so very few iterations are needed on fine levels, where computation is expensive. NI strategies have been applied successfully to a variety of applications that involve coupled physics (e.g. $[4,6,12,37])$.

To demonstrate the utility of deflation combined with NI and multigrid, we consider a variety of static liquid crystal problems. The presence of multiple solutions to liquid crystal systems is well-known and central to their use in many industries, including modern display technology. Numerical simulations of liquid crystal configurations are used to examine theory, explore new physical phenomena $[1,7]$, and optimize device performance. For static liquid crystal structures, the associated system of PDEs, known as the equilibrium equations [20,46], permit multiple solutions, even under relatively mild complexity [18]. Further, multiple locally optimal configurations and saddle-point structures may exist for the energy formulation. Thus, in this paper, we incorporate deflation into the constrained optimization approach previously developed in $[1,2]$. This yields an adaptable and efficient method that enables the discovery of distinct solutions for such problems.

This paper is organized as follows. The deflation technique is discussed and derived in the context of variational optimization formulations in Section 2. The combination of deflation with NI and the integration of existing (multigrid) preconditioners is also examined in this section. The liquid crystal energy model and existing minimization approach without deflation are summarized in Section 3. In Sections 4 and 5 , the implementation of the algorithm is outlined and four numerical experiments are performed. Finally, Section 6 provides some concluding remarks and a discussion of future work.

2. Deflation methodology. A standard but unsystematic approach to computing distinct solutions for nonlinear problems with several solutions is the use of numerous initial guesses as part of an overarching Newton-type scheme, known as multistart methods [38]. In this section, we adapt the deflation technique first pro- 
posed in [22] as a more effective and systematic alternative. The essential idea is to cast the constrained minimization problem as the solution of the associated optimality conditions and then to repeatedly modify the nonlinear problem to eliminate known solutions as they are found.

First, consider a general variational problem, posed as $\min _{\mathbf{u} \in U} \mathcal{L}(\mathbf{u})$, where $\mathbf{u} \in U$ gathers the continuum variables to be solved for, and $\mathcal{L}: U \rightarrow \mathbb{R}$ is a nonlinear functional. Then, the Euler-Lagrange equations are naturally given by the first variation, such that

$$
\mathcal{A}(\mathbf{u} ; \mathbf{v}):=\mathcal{L}_{\mathbf{u}}[\mathbf{v}]=0 \quad \forall \mathbf{v} \in U
$$

where the Gâteaux derivative, $\mathcal{L}_{\mathbf{u}}[\mathbf{v}]$, is evaluated at $\mathbf{u}$ in the direction $\mathbf{v}$ (when imposing Dirichlet boundary conditions on $\mathbf{u}$, we may also take $\mathbf{v}$ in $U_{0}$, corresponding to the test space appropriately adjusted to functions that take value 0 on the Dirichlet boundary). Note that $\mathcal{A}: U \times U \rightarrow \mathbb{R}$ is linear in its second argument and can be written as $\mathcal{A}(\mathbf{u} ; \mathbf{v})=\langle\mathbf{f}(\mathbf{u}), \mathbf{v}\rangle$, where $\mathbf{f}: U \rightarrow U^{*}$, and $U^{*}$ is the dual space of $U$.

We think of the nonlinear equation to be solved as either the variational form in (2.1) or the "residual" equation $\mathbf{f}(\mathbf{u})=\mathbf{0}$ (noting that this equality is taken to be in $\left.U^{*}\right)$, and note that it may have multiple solutions, even when there is a unique global minimum to the original variational problem. The question of whether computed solutions to the Euler-Lagrange equations represent global minima or only local minima (or maxima or saddle points) is often difficult to answer with certainty. The deflation technique presented in this section systematically promotes the discovery of numerous solutions, revealing stable and unstable local minima and increasing the probability of the identification of a global minimizer.

Suppose one solution, $\mathbf{r}$, to (2.1) has been found. We construct a deflated residual

$$
\mathbf{g}(\mathbf{u})=M_{p, \alpha}(\mathbf{u} ; \mathbf{r}) \mathbf{f}(\mathbf{u}),
$$

where $M_{p, \alpha}$ is the shifted deflation operator,

$$
M_{p, \alpha}(\mathbf{u} ; \mathbf{r})=\left(\frac{1}{\|\mathbf{u}-\mathbf{r}\|_{U}^{p}}+\alpha\right) \mathbf{I}
$$

Here $\alpha \geq 0$ is a scalar shift, $p \in[1, \infty)$ is the deflation exponent, and $\mathbf{I}$ is the identity operator on $U^{*}$. Thus, for fixed $\mathbf{u}, \mathbf{r} \in U$, the deflation operator $M_{p, \alpha}(\mathbf{u} ; \mathbf{r})$ is simply a scaled identity operator in $U^{*}$. Applying the deflation operator to the residual $\mathbf{f}$ ensures that Newton's method applied to the resulting variational system will not converge to $\mathbf{r}$ under mild regularity conditions on the solution [22]. In the context of (2.1), the resulting deflated variational operator is given by

$$
\mathcal{G}(\mathbf{u} ; \mathbf{v})=\left\langle M_{p, \alpha}(\mathbf{u} ; \mathbf{r}) \mathbf{f}(\mathbf{u}), \mathbf{v}\right\rangle=\left\langle\left(\frac{1}{\|\mathbf{u}-\mathbf{r}\|_{U}^{p}}+\alpha\right) \mathbf{f}(\mathbf{u}), \mathbf{v}\right\rangle .
$$

This produces the deflated variational problem

$$
\mathcal{G}(\mathbf{u} ; \mathbf{v})=0 \quad \forall \mathbf{v} \in U .
$$

A nonzero shift, $\alpha$, is applied so that the deflated residual does not tend to zero as $\|\mathbf{u}-\mathbf{r}\|_{U}$ becomes arbitrarily large, see [22]. While the method is generally robust with respect to parameter choice, there are situations where additional performance improvements are attainable for certain selections of $p$ and $\alpha$. 
For brevity, we suppress the semicolon notation in the variational operators except when necessary for clarity and denote $\eta(\mathbf{u})=\left(\frac{1}{\|\mathbf{u}-\mathbf{r}\|_{U}^{p}}+\alpha\right)$. Note that the deflated residual $\mathbf{g}(\mathbf{u})=\eta(\mathbf{u}) \mathbf{f}(\mathbf{u})$, is also nonlinear. Thus, we consider its solution via Newton's method, linearizing $\mathbf{g}(\mathbf{u})=0$ around an approximate solution, $\mathbf{u}_{k}$, and solving for an update $\delta \mathbf{u}$ that satisfies

$$
\left\langle\mathbf{g}\left(\mathbf{u}_{k}\right)+\mathbf{g}^{\prime}\left(\mathbf{u}_{k}\right) \delta \mathbf{u}, \mathbf{v}\right\rangle=0 \quad \forall \mathbf{v} \in U,
$$

where $\mathbf{g}^{\prime}\left(\mathbf{u}_{k}\right)$ is the Fréchet derivative of $\mathbf{g}(\mathbf{u})$ evaluated at $\mathbf{u}_{k}$. Applying the product rule to $\mathbf{g}(\mathbf{u})$, we have that

$$
\mathbf{g}^{\prime}\left(\mathbf{u}_{k}\right)=\eta\left(\mathbf{u}_{k}\right) \mathbf{f}^{\prime}\left(\mathbf{u}_{k}\right)+\mathbf{f}\left(\mathbf{u}_{k}\right) \eta^{\prime}\left(\mathbf{u}_{k}\right)
$$

where $\mathbf{f}^{\prime}\left(\mathbf{u}_{k}\right)$ and $\eta^{\prime}\left(\mathbf{u}_{k}\right)$ are the Fréchet derivatives of $\mathbf{f}$ and $\eta$, respectively, evaluated at $\mathbf{u}_{k}$. We note that $\mathbf{g}^{\prime}\left(\mathbf{u}_{k}\right)$ and $\mathbf{f}^{\prime}\left(\mathbf{u}_{k}\right)$ become the standard Jacobians of $\mathbf{g}$ and $\mathbf{f}$ when discretized, as discussed below. With this, we now discretize and solve the linearized variational problem: find $\delta \mathbf{u}$ such that

$$
\left\langle\mathbf{g}^{\prime}\left(\mathbf{u}_{k}\right) \delta \mathbf{u}, \mathbf{v}\right\rangle=-\left\langle\mathbf{g}\left(\mathbf{u}_{\mathbf{k}}\right), \mathbf{v}\right\rangle \quad \forall \mathbf{v} \in U .
$$

This is used to compute the updated approximation $\mathbf{u}_{k+1}=\mathbf{u}_{k}+\omega \delta \mathbf{u}$ with a step length $\omega \in \mathbb{R}$.

2.1. Deflated linear systems. A strategy for constructing effective preconditioners for the deflated system based on existing preconditioners for the undeflated matrices and computing their actions in a matrix-free fashion is presented in [22]. This section provides a general framework for efficient reuse of good preconditioners designed for the original Newton linearizations, particularly necessary in the context of mixed finite-element discretizations of multiphysics problems. In Sections 4 and 5, we utilize this approach to apply monolithic multigrid preconditioners, as developed in $[1,3]$, to solve for the updates in (2.4).

Let $F\left(\mathbf{u}_{k}\right)$ and $G\left(\mathbf{u}_{k}\right)$ denote the vectors corresponding to discretizations of $\mathbf{f}\left(\mathbf{u}_{k}\right)$ and $\mathbf{g}\left(\mathbf{u}_{k}\right)$, respectively, and let $d\left(\mathbf{u}_{k}\right)$ be the discretization vector corresponding to the Fréchet derivative of $\eta$. Let $J_{G}\left(\mathbf{u}_{k}\right)$ and $J_{F}\left(\mathbf{u}_{k}\right)$ indicate the discretized Jacobians of the deflated and undeflated systems, respectively. Then,

$$
J_{G}\left(\mathbf{u}_{k}\right)=\eta\left(\mathbf{u}_{k}\right) J_{F}\left(\mathbf{u}_{k}\right)+F\left(\mathbf{u}_{k}\right) d\left(\mathbf{u}_{k}\right)^{T} .
$$

As defined, $J_{G}\left(\mathbf{u}_{k}\right)$ is composed of a rank-one update to $J_{F}\left(\mathbf{u}_{k}\right)$. Thus, $J_{G}\left(\mathbf{u}_{k}\right)$ is generally dense, even if $J_{F}\left(\mathbf{u}_{k}\right)$ is not, and explicit construction and computation with the matrix is prohibitively expensive. Throughout the remainder of the paper, except when necessary for clarity, we neglect the dependence on $\mathbf{u}_{k}$ in the notation.

Considering $J_{G}=\left(\eta J_{F}+F d^{T}\right)$ and applying the Sherman-Morrison formula [30] gives

$$
J_{G}^{-1}=\left(\eta J_{F}+F d^{T}\right)^{-1}=\frac{J_{F}^{-1}}{\eta}-\frac{\frac{1}{\eta^{2}} J_{F}^{-1} F d^{T} J_{F}^{-1}}{1+\frac{1}{\eta} d^{T} J_{F}^{-1} F} .
$$

Using (2.6) to compute the discretized update vector corresponding to (2.4) produces

$$
\begin{aligned}
-J_{G}^{-1} G=-\frac{J_{F}^{-1} G}{\eta}+\frac{\frac{1}{\eta^{2}} J_{F}^{-1} F d^{T} J_{F}^{-1} G}{1+\frac{1}{\eta} d^{T} J_{F}^{-1} F} & =-J_{F}^{-1} F+\frac{\frac{1}{\eta} J_{F}^{-1} F d^{T} J_{F}^{-1} F}{1+\frac{1}{\eta} \cdot d^{T} J_{F}^{-1} F} \\
& =-\left(1-\frac{\frac{1}{\eta} \cdot d^{T} J_{F}^{-1} F}{1+\frac{1}{\eta} \cdot d^{T} J_{F}^{-1} F}\right) J_{F}^{-1} F
\end{aligned}
$$


Note that $J_{F}^{-1} F$ corresponds to assembling and solving the original undeflated problem and $d^{T} J_{F}^{-1} F$ is a dot product resulting in a scalar. Thus, solving the discrete form of the deflation system in (2.4) is reduced to a single solve with the original sparse system, one dot product, one vector scaling, and a few scalar operations. Therefore, any preconditioner developed to effectively solve the undeflated Newton systems can be directly applied to the deflated systems that arise in (2.4), yielding an efficient algorithm for computing the deflated updates.

2.2. Multiple deflation. Thus far, the class of deflation operators considered focuses on deflation with one known solution, $\mathbf{r}$. In this section, we briefly discuss extending the deflation procedure to treat a family of known solutions $\mathbf{r}_{1}, \mathbf{r}_{2}, \ldots, \mathbf{r}_{m}$. With several known solutions, the multiple deflation operator is the product of the single deflation operators for each individual solution such that

$$
M_{p, \alpha}\left(\mathbf{u} ; \mathbf{r}_{1}, \mathbf{r}_{2}, \ldots, \mathbf{r}_{m}\right)=\prod_{i=1}^{m} M_{p, \alpha}\left(\mathbf{u} ; \mathbf{r}_{i}\right)
$$

This modifies the action of $M_{p, \alpha}\left(\mathbf{u} ; \mathbf{r}_{1}, \mathbf{r}_{2}, \ldots, \mathbf{r}_{m}\right)$ on $\mathbf{f}(\mathbf{u})$ such that

$$
\mathcal{G}(\mathbf{u} ; \mathbf{v})=\left\langle\prod_{i=1}^{m} M_{p ; \alpha}\left(\mathbf{u}, \mathbf{r}_{i}\right) \mathbf{f}(\mathbf{u}), \mathbf{v}\right\rangle=\left\langle\left(\prod_{i=1}^{m}\left(\frac{1}{\left\|\mathbf{u}-\mathbf{r}_{i}\right\|_{U}^{p}}+\alpha\right)\right) \mathbf{f}(\mathbf{u}), \mathbf{v}\right\rangle,
$$

which we recognize, as in the case of single deflation, to be a scaling of the residual with the form $\mathbf{g}(\mathbf{u})=\eta(\mathbf{u}) \mathbf{f}(\mathbf{u})$. This deflated system remains nonlinear and corresponding linearizations are derived to compute distinct solutions satisfying the first-order optimality conditions. As with the single deflation linearization, the discretized multiple deflation Jacobian, $J_{G}$, is composed of a rank-one update to $J_{F}$ as in (2.5), though $d\left(\mathbf{u}_{k}\right)$ is now more complicated than the single deflation case. A process similar to that applied in the single deflation case reveals an analogous result for computation of solutions to the discretized, deflated linearizations and yields similar results enabling the application of preexisting preconditioners to linear systems subject to deflation over several known solutions. Each of the simulations to follow employs multi-solution deflation operators as distinct solutions are discovered.

2.3. Interaction with nested iteration. To improve the efficiency of the overall algorithm, we propose here to directly integrate the deflation methodology with NI. The process begins on a coarse grid, with a small number of degrees of freedom, followed by a series of refinements until the final desired mesh resolution is obtained. On each refinement level, a combination of continued iteration on known solutions followed by applying deflation to uncover additional solutions is performed. The general numerical flow is detailed below in Algorithm 1. The algorithm has four main stages. The outermost phase is an NI hierarchy that has proven highly effective in reducing computational work for systems of nonlinear PDEs discretized by appropriate finite-element methods $[2,4-6,12,37,44]$. On each mesh, the algorithm first performs (undeflated) Newton iterations on interpolated versions of solutions found on the previous, coarser mesh, termed the continuation list in Algorithm 1, to further resolve the solution features on the finer mesh. On the coarsest mesh, one or more simple initial guesses are taken in place of the interpolated information. This procedure is followed by a solution discovery stage where all known solutions are deflated and new 
solutions are sought. Each deflation solve begins with an initial guess taken from a list of (possibly several) initialization vectors. Newton iterations are performed until a convergence tolerance is reached for a new solution (added to the solution list in Algorithm 1) or a maximum number of Newton iterations have been performed. For both the deflated and undeflated Newton iterations, the convergence stopping criterion on a given level is based on a set tolerance for an approximation's conformance to the first-order optimality conditions in the standard Euclidean $l^{2}$-norm. Throughout the numerical results section below, this tolerance is held at $10^{-4}$. Since this is imposed in the discrete $l^{2}$-norm, this translates to an increasingly tighter constraint on the error in the continuum $L^{2}$-norm as the mesh is refined. For each deflated Newton iteration, the matrix-free approach outlined in Section 2.1 can be used, so that the efficiency of the linear iterations depends only on that of the underlying preconditioner (or other solver) for the undeflated system. Finally, the known solution approximations are interpolated to a finer grid to form the continuation list there. In the current implementation, these finer grids represent successive uniform refinements of the initial coarse grid.

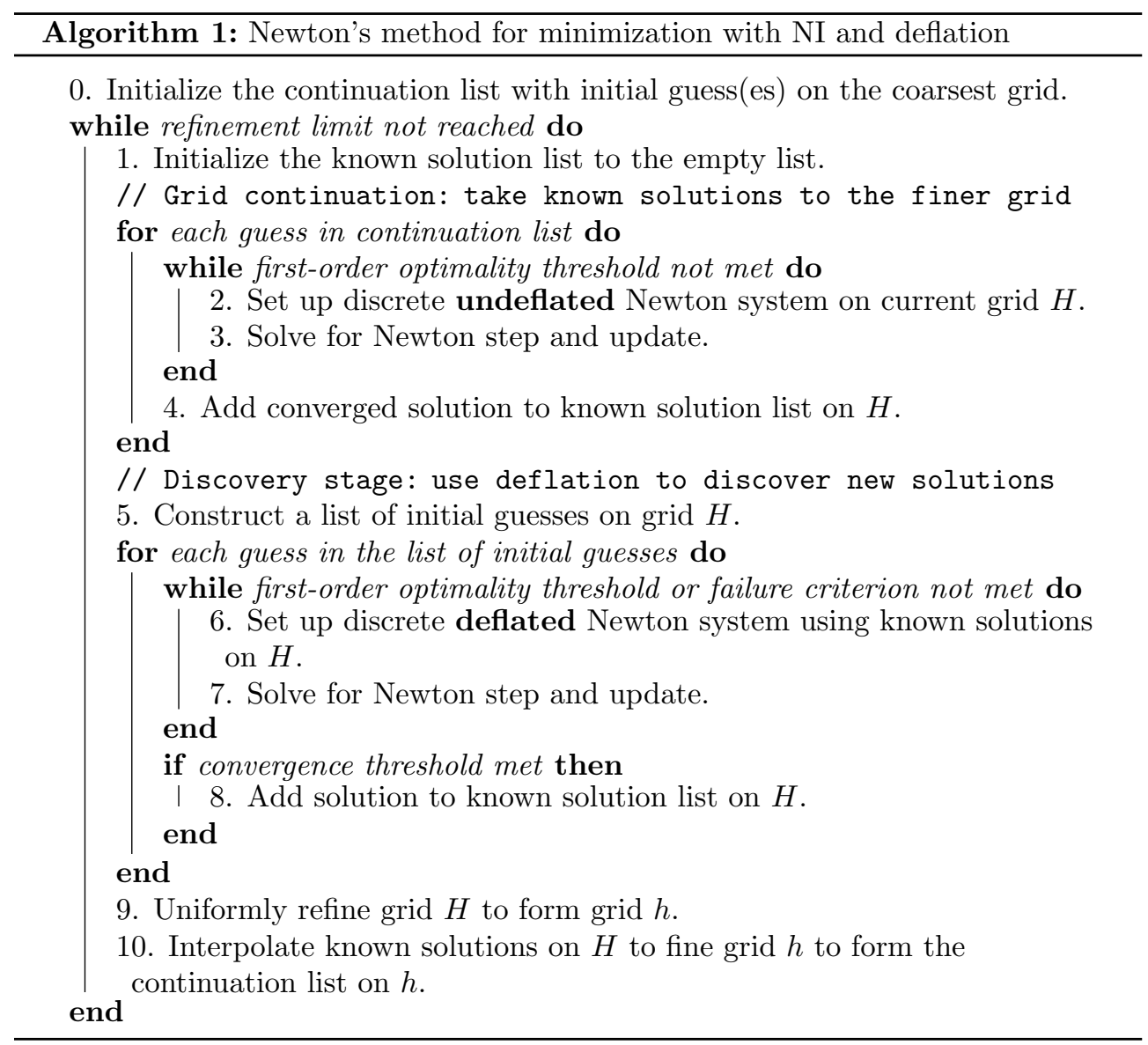

The blending of NI and deflation outlined above has a number of advantages above and beyond efficiency. Certain solutions are more readily detectable through deflation processes on both coarser and finer meshes, as observed in the numerical results to 
follow. Moreover, the algorithm allows for varying and adaptive initial guesses. That is, in addition to a static set of initial guesses for the deflation solves on each grid, sets of initial guesses may be constructed from transformations of known or newly discovered solutions throughout the NI and deflation process. Constructing strategies for adaptive generation of initial guesses will be the subject of future work.

2.4. Newton stepping. A form of damped Newton stepping is applied for both the undeflated and deflated updates such that the next iterates are given by

$$
\mathbf{u}_{k+1}=\mathbf{u}_{k}+\omega \delta \mathbf{u}
$$

where $0<\omega \leq 1$ is a damping parameter. For the undeflated solves, we take $\omega=\omega_{1}$ on the coarsest grid and increase its value by $\Delta_{1}$ at each level of refinement to a maximum value of 1 . With the deflated systems, we take $\omega=\omega_{2}$ on the coarsest grid and decrease its value by $\Delta_{2}$ at each level of refinement to a minimum value of 0.2 . This strategy aims at improving convergence for both types of iterations. For the undeflated solves, the damping parameter is increased on each grid as confidence in the Newton convergence increases for more finely resolved solutions. On the other hand, in numerical experiments, some convergence issues have been observed for deflation iterations beginning from poor initial guess on fine meshes. Hence, the decreasing damping parameter increases the likelihood of convergence on finer grids within the deflation iterations. While more advanced step selection methods, such as trust regions [5,10], are well-established, we experimentally observed that using trust regions during the deflation phase of the algorithm hindered the method's ability to discover new basins of attraction, thereby limiting the number of unique solutions found. Specifically, with the application of the deflation operator, additional nonlinearity is introduced to the variational system under consideration. This generally results in small trust regions, and thereby significant Newton damping, especially in regions near previously known solutions. In this way, progress towards undiscovered solutions can be exceedingly slow or even stall. Improving this performance and investigating alternate approaches to automatic step parameter selection will be considered in future work.

3. Variational modeling of nematic liquid crystals. To demonstrate the effectiveness of Algorithm 1, we consider variational models of nematic liquid crystals, as previously discussed in $[1,2,5]$. In those papers, the focus was on efficiently identifying a single solution to the Euler-Lagrange equations derived from the nonlinear variational problem, aiming (but not guaranteeing) to find the global minimizer. Here, the goal is to directly identify multiple solutions using the combined deflation-NI algorithm. In this section, we review the variational model of nematic liquid crystals previously studied in $[1,2,5]$, for which numerical results are presented in Section 4.

Liquid crystals are materials with mesophases exhibiting characteristics of both liquids and structured solids. These mesophases are found at varying temperatures and solvent concentrations and exist for many types of materials, including both synthetic [27] and naturally occurring molecular compositions [14]. They permit a striking variety of arrangements and behaviors. In this paper we focus on two: nematic phases, which consist of rod-like molecules, and cholesteric liquid crystals, which share many similarities with nematics but intrinsically prefer helical structures that admit less symmetry due to chiral preference. These types of liquid crystals self-assemble into ordered structures characterized by a preferred average direction at each point known as the director. The director is described by a unit vector field at each point 
and is denoted $\mathbf{n}(x, y, z)=\left(n_{1}(x, y, z), n_{2}(x, y, z), n_{3}(x, y, z)\right)^{T}$. Along with their crystalline self-structuring, liquid crystals demonstrate a number of important physical phenomena including birefringence, electric coupling, and flexoelectric effects. Comprehensive reviews of liquid crystal physics are found in $[17,46,47]$. These properties and others have led to many important discoveries and a diversity of applications (e.g. $[33,43,48])$.

3.1. Frank-Oseen free-energy model. While a number of models exist [16, 40,46], we consider the Frank-Oseen free-energy model for the computation of liquid crystal equilibrium configurations $[46,47]$. The complexity of the model and the necessary nonlinear pointwise unit-length constraint have limited the availability of analytical solutions in the absence of significant simplifying assumptions. Recently, a number of numerical methods $[7,26,41,42]$ have been proposed for the Frank-Oseen model. In $[1,2]$, an energy-minimization finite-element technique was developed that allows accurate and efficient computational simulation of liquid crystal behavior. This approach is presented here and combined with the deflation-NI methodology below.

The Frank-Oseen free-energy model characterizes the equilibrium free energy for a domain $\Omega$ by deformations of the nondimensional, unit-length director field, $\mathbf{n}$. Liquid crystals tend towards configurations exhibiting minimal free energy. Let $K_{i}, i=1,2,3$, be the Frank constants [23] with $K_{i} \geq 0$ [21]. Here, we consider the case that each $K_{i} \neq 0$. These constants are often anisotropic (i.e., $K_{1} \neq K_{2} \neq K_{3}$ ), vary with liquid crystal type, and play important roles in physical phenomena $[8,34]$. In order to properly formulate the Lagrangian below, a nondimensionalization, introduced in [5], using a characteristic length scale, $\sigma$, a characteristic Frank constant, $K$, and a characteristic voltage, $\phi_{0}>0$, is applied so that the entire expression is dimensionless.

We denote the classical $L^{2}(\Omega)$ inner product and norm as $\langle\cdot, \cdot\rangle_{0}$ and $\|\cdot\|_{0}$, respectively, for both scalar and vector quantities. Throughout this paper, we assume the presence of Dirichlet boundary conditions or mixed Dirichlet and periodic boundary conditions on a rectangular domain and, therefore, utilize the null Lagrangian simplification discussed in $[1,46]$. Hence, including the possibility of external electric fields (but not flexoelectric effects), the Frank-Oseen free energy for nematics is written as

$$
\begin{gathered}
\mathcal{F}(\mathbf{n}, \phi)=K_{1}\|\nabla \cdot \mathbf{n}\|_{0}^{2}+K_{3}\langle\mathbf{Z} \nabla \times \mathbf{n}, \nabla \times \mathbf{n}\rangle_{0}-\epsilon_{0} \epsilon_{\perp}\langle\nabla \phi, \nabla \phi\rangle_{0} \\
-\epsilon_{0} \epsilon_{a}\langle\mathbf{n} \cdot \nabla \phi, \mathbf{n} \cdot \nabla \phi\rangle_{0},
\end{gathered}
$$

where $\phi$ is an electric potential, $\epsilon_{0}$ denotes the permittivity of free space, and the dimensionless constants $\epsilon_{\perp}$ and $\epsilon_{a}$ are the perpendicular dielectric permittivity and dielectric anisotropy of the liquid crystal, respectively. Finally, $\mathbf{Z}=\kappa \mathbf{n} \otimes \mathbf{n}+(\mathbf{I}-$ $\mathbf{n} \otimes \mathbf{n})=\mathbf{I}-(1-\kappa) \mathbf{n} \otimes \mathbf{n}$, is a dimensionless tensor, where $\kappa=K_{2} / K_{3}$. Note that if $\kappa=1, \mathbf{Z}$ reduces to the identity. In the existing literature (including [1,2]), the functional in (3.1) is typically scaled by a factor of $\frac{1}{2}$; while we derive the expressions below without this scaling, we include it in the free energies reported in Sections 4 and 5 for consistency with other papers.

The director field is subject to a local unit-length constraint such that $\mathbf{n} \cdot \mathbf{n}=1$ at each point throughout the domain. In [5], numerical evidence indicates that imposing this constraint with Lagrange multipliers is an accurate and highly efficient approach, particularly in comparison to penalty or renormalization formulations. Note that in the case that $K_{1}=K_{2}=K_{3}$ with no electric field, a linear free-energy functional is obtained. Coupled with the unit-length constraint, the minimization corresponds to a weak harmonic mapping problem. Existence and uniqueness theory for this type of problem has been analyzed in [31]. 
Throughout this paper, we will make use of the spaces $H(\operatorname{div}, \Omega)=\{\mathbf{w} \in$ $\left.\left(L^{2}(\Omega)\right)^{3}: \nabla \cdot \mathbf{w} \in L^{2}(\Omega)\right\}$ and $H(\operatorname{curl}, \Omega)=\left\{\mathbf{w} \in\left(L^{2}(\Omega)\right)^{3}: \nabla \times \mathbf{w} \in\left(L^{2}(\Omega)\right)^{3}\right\}$. As in [1], define

$$
\mathcal{H}^{D C}(\Omega)=\{\mathbf{w} \in H(\operatorname{div}, \Omega) \cap H(\operatorname{curl}, \Omega): B(\mathbf{w})=\overline{\mathbf{g}}\},
$$

with norm $\|\mathbf{w}\|_{D C}^{2}=\|\mathbf{w}\|_{0}^{2}+\|\nabla \cdot \mathbf{w}\|_{0}^{2}+\|\nabla \times \mathbf{w}\|_{0}^{2}$ and appropriate boundary conditions $B(\mathbf{w})=\overline{\mathbf{g}}$. Here, we assume that $\overline{\mathbf{g}}$ satisfies appropriate compatibility conditions for the operator $B$. For example, if $B$ represents full Dirichlet boundary conditions and $\Omega$ has a Lipschitz continuous boundary, it is assumed that $\overline{\mathbf{g}} \in H^{\frac{1}{2}}(\partial \Omega)^{3}$ [28]. Further, let $\mathcal{H}_{0}^{D C}(\Omega)=\{\mathbf{w} \in H(\operatorname{div}, \Omega) \cap H(\operatorname{curl}, \Omega): B(\mathbf{w})=\mathbf{0}\}$. Note that if $\Omega$ is a Lipschitz domain and $B$ imposes full Dirichlet boundary conditions on all components of $\mathbf{w}$, then $\mathcal{H}_{0}^{D C}(\Omega)=\left(H_{0}^{1}(\Omega)\right)^{3}[28$, Lemma 2.5]. Denote

$$
H^{1, g}(\Omega)=\left\{f \in H^{1}(\Omega): B_{1}(f)=g\right\},
$$

where $H^{1}(\Omega)$ represents the classical Sobolev space with norm $\|\cdot\|_{1}$ and $B_{1}(f)=g$ is an appropriate boundary condition expression for the electric potential, $\phi$.

We define the Lagrangian as

$$
\mathcal{L}(\mathbf{n}, \phi, \lambda)=\mathcal{F}(\mathbf{n}, \phi)+\int_{\Omega} \lambda(\mathbf{x})(\mathbf{n} \cdot \mathbf{n}-1) d V,
$$

where $\mathcal{L}(\mathbf{n}, \phi, \lambda)$ has been nondimensionalized in the same fashion as the free-energy functional. To minimize the functional, first-order optimality conditions are derived as

$$
\begin{array}{rlrl}
\mathcal{L}_{\mathbf{n}}[\mathbf{w}] & =\frac{\partial}{\partial \mathbf{n}} \mathcal{L}(\mathbf{n}, \phi, \lambda)[\mathbf{w}]=0 & & \forall \mathbf{w} \in \mathcal{H}_{0}^{D C}(\Omega), \\
\mathcal{L}_{\phi}[\psi]=\frac{\partial}{\partial \phi} \mathcal{L}(\mathbf{n}, \phi, \lambda)[\psi]=0 & & \forall \psi \in H^{1,0}(\Omega), \\
\mathcal{L}_{\lambda}[\gamma]=\frac{\partial}{\partial \lambda} \mathcal{L}(\mathbf{n}, \phi, \lambda)[\gamma]=0 & & \forall \gamma \in L^{2}(\Omega),
\end{array}
$$

with $\mathcal{A}(\mathbf{n}, \phi, \lambda ; \mathbf{w}, \psi, \gamma)=\mathcal{L}_{\mathbf{n}}[\mathbf{w}]+\mathcal{L}_{\phi}[\psi]+\mathcal{L}_{\lambda}[\gamma]$ being the combined variational form. For the Frank-Oseen model in (3.1), these Gâteaux derivatives are given by

$$
\begin{aligned}
\mathcal{L}_{\mathbf{n}}[\mathbf{w}]=2 K_{1}\langle\nabla \cdot \mathbf{n}, \nabla \cdot \mathbf{w}\rangle_{0}+2 K_{3}\langle\mathbf{Z} \nabla \times \mathbf{n}, \nabla \times \mathbf{w}\rangle_{0} & & \\
\quad+2\left(K_{2}-K_{3}\right)\langle\mathbf{n} \cdot \nabla \times \mathbf{n}, \mathbf{w} \cdot \nabla \times \mathbf{n}\rangle_{0} & & \\
\quad-2 \epsilon_{0} \epsilon_{a}\langle\mathbf{n} \cdot \nabla \phi, \mathbf{w} \cdot \nabla \phi\rangle_{0}+2 \int_{\Omega} \lambda(\mathbf{n}, \mathbf{w}) d V=0, & & \forall \mathbf{w} \in \mathcal{H}_{0}^{D C}(\Omega), \\
\mathcal{L}_{\phi}[\psi]=-2 \epsilon_{0} \epsilon_{\perp}\langle\nabla \phi, \nabla \psi\rangle_{0}-2 \epsilon_{0} \epsilon_{a}\langle\mathbf{n} \cdot \nabla \phi, \mathbf{n} \cdot \nabla \psi\rangle_{0}=0, & & \forall \psi \in H^{1,0}(\Omega), \\
\mathcal{L}_{\lambda}[\gamma]=\int_{\Omega} \gamma((\mathbf{n}, \mathbf{n})-1) d V=0, & & \forall \gamma \in L^{2}(\Omega) .
\end{aligned}
$$

To express this problem in the general framework of Section 2, we identify the product space $U=\mathcal{H}^{D C}(\Omega) \times H^{1, g}(\Omega) \times L^{2}(\Omega)$ with associated norm $\|\cdot\|_{U}$, and its subspace $U_{0}=\mathcal{H}_{0}^{D C}(\Omega) \times H^{1,0}(\Omega) \times L^{2}(\Omega)$. For any $\mathbf{u} \in U$, we naturally identify $\mathbf{u}=$ $(\mathbf{n}, \phi, \lambda)^{T}$. The Euler-Lagrange equations above in (3.2)-(3.4) define the variational form $\mathcal{A}: U \times U_{0} \rightarrow \mathbb{R}$ termwise, giving the first variation as finding $\mathbf{u} \in U$ such that $\mathcal{A}(\mathbf{u} ; \mathbf{v})=0$ for all $\mathbf{v} \in U_{0}$. Note that this variational system is nonlinear and, in 
many cases, admits several distinct solutions. For example, the classical Freedericksz transition problem [24,49], which is discussed in detail below, admits at least three solutions to the first-order optimality conditions.

In $[1,2]$, Newton linearizations and a finite-element discretization are used to compute solutions to this variational system. This linearization yields the Newton update equations

$$
J_{F}\left(\mathbf{u}_{k}\right)[\delta \mathbf{u}]=\left[\begin{array}{ccc}
\mathcal{L}_{\mathbf{n n}} & \mathcal{L}_{\mathbf{n} \phi} & \mathcal{L}_{\mathbf{n} \lambda} \\
\mathcal{L}_{\phi \mathbf{n}} & \mathcal{L}_{\phi \phi} & \mathbf{0} \\
\mathcal{L}_{\lambda \mathbf{n}} & \mathbf{0} & \mathbf{0}
\end{array}\right]\left[\begin{array}{c}
\delta \mathbf{n} \\
\delta \phi \\
\delta \lambda
\end{array}\right]=-\left[\begin{array}{c}
\mathcal{L}_{\mathbf{n}} \\
\mathcal{L}_{\phi} \\
\mathcal{L}_{\lambda}
\end{array}\right]
$$

where each of the system components is evaluated at $\mathbf{n}_{k}, \phi_{k}$, and $\lambda_{k}$, the current approximations for $\mathbf{n}, \phi$, and $\lambda$, and $\delta \mathbf{n}=\mathbf{n}_{k+1}-\mathbf{n}_{k}, \delta \phi=\phi_{k+1}-\phi_{k}$, and $\delta \lambda=$ $\lambda_{k+1}-\lambda_{k}$ are the updates we seek to compute. Here, following the mixed finiteelement formulation in [1,2], we write $J_{F}$ as a matrix acting on the components of $\delta \mathbf{u}=(\delta \mathbf{n}, \delta \phi, \delta \lambda)^{T}$, rather than in the more compact notation of Section 2. For the full Hessian computations, see Appendix A. In addition to enabling the construction of the linearized system, deriving the components of the Hessian permits numerical study of the energetic stability of computed solutions. Techniques for such analysis are not developed here but are possible as a post-processing step once equilibrium states have been computed.

3.2. Discretization and multigrid preconditioning of the Jacobian system. For the test problems below, we consider a classical domain with two parallel substrates placed at unit distance apart. These substrates run parallel to the $x z$-plane and perpendicular to the $y$-axis. Further, we assume a slab-type domain such that $\mathbf{n}$ may have a non-zero $z$-component, but $\frac{\partial \mathbf{n}}{\partial z}=\mathbf{0}$. Thus, for the numerical experiments to follow, $\Omega=\{(x, y): 0 \leq x, y \leq 1\}$. In the first two experiments of Section 4, periodic boundary conditions are applied at the left and right boundaries and Dirichlet conditions are enforced at the top and bottom of the domain. In the third experiment of the section, Dirichlet boundary conditions are applied for the entire boundary. In all experiments, we use biquadratic finite elements to discretize components associated with $\mathbf{n}$ and $\phi$ in the variational systems, while piecewise constants are used for those related to $\lambda$. In each simulation, the algorithm begins on a uniform $8 \times 8$ coarse mesh, ascending in uniform refinements to a $256 \times 256$ fine grid. This results in a total of 1,118, 212 degrees of freedom on the finest mesh. The algorithm's discretizations and grid management are performed with the deal.II scientific computing library [9].

For preconditioning of the GMRES linear solver applied in the numerical simulations, we employ a monolithic multigrid approach previously developed in $[1,3,5]$. This approach uses geometric multigrid, taking advantage of the structured grids used in the discretization described above; grid-transfer operators are defined based on the standard finite-element interpolation operators, while coarse-grid operators are defined via Galerkin coarsening. The relaxation scheme used is a modified scheme from the Braess-Sarazin family, defined by an approximate block factorization of the system matrix. In the results below, we use a standard multigrid V-cycle with a single pre- and post-relaxation sweep.

4. Numerical results for nematic liquid crystals. In this section and the next, four numerical experiments using the combined deflation and NI approach, detailed in Section 2, are carried out to demonstrate the performance of the method. The first two simulations consider problems with known analytical solutions. The 
remaining experiments illustrate the full capabilities of the algorithm. For each simulation, the characteristic length scale discussed above is taken to be one micron, such that $\sigma=10^{-6} \mathrm{~m}$. Furthermore, the characteristic Frank constant is taken to be $K=6.2 \times 10^{-12} \mathrm{~N}$, the dimensional value of $K_{1}$ for $5 \mathrm{CB}$, a common liquid crystal. The applied nondimensionalization, for instance, yields parameters $K_{1}=1, K_{2}=0.62903$, and $K_{3}=1.32258$ for $5 \mathrm{CB}$. In addition, the characteristic voltage is $\phi_{0}=1 \mathrm{~V}$, which implies that the nondimensional dielectric permittivity constant is $\epsilon_{0}=1.42809$.

Unless otherwise stated, the deflation parameters are fixed such that $\alpha=1$ and $p=3$, and the failure criterion in Algorithm 1 occurs when the number of Newton iterations reaches 100 (without convergence) or the average length of the current director field is above 3, substantially violating the unit-length constraint. (Similar failure criteria could be integrated with the first Newton loop in Algorithm 1, for continuing known solutions, but this appears to be unnecessary in practice.) The linear solver tolerance, which is based on a ratio of the norm of the current (discrete) solution's residual to that of the initial guess, is held at $10^{-6}$.

Computational work for a full NI solve is given in terms of work units (WUs), calculated as a weighted sum of the total number of V-cycles across each NI level. With uniform mesh refinements and a geometric multigrid strategy, the total number of $\mathrm{V}$-cycles on each grid is weighted by $(1 / 4)^{l}$, where $l$ is the level of coarsening away from the finest mesh. For example, the total number of V-cycles on the second finest mesh is simply scaled by $1 / 4$. Thus, the total WUs for a given NI solve provides a work measurement equivalent to counting fine-grid V-cycles in a single-grid approach.

4.1. Tilt-twist configuration. The first problem considered in this section is an elastic configuration with no electric field and Frank constants given by $K_{1}=1.0$, $K_{2}=3.0$, and $K_{3}=1.2$. For the Newton damping, $\omega_{1}=1.0, \Delta_{1}=0.0, \omega_{2}=1.0$, and $\Delta_{2}=0.5$. At the Dirichlet boundaries, we set

$$
\mathbf{n}(x, 0)=\left(\cos \left(-\frac{\pi}{4}\right), 0, \sin \left(-\frac{\pi}{4}\right)\right), \quad \mathbf{n}(x, 1)=\left(\cos \left(\frac{\pi}{4}\right), 0, \sin \left(\frac{\pi}{4}\right)\right) .
$$

This is known as a tilt-twist problem and is an interesting example for a few reasons. The opposing boundary conditions induce a twisting configuration in the nematics through the interior of the domain. Under these conditions, a planar twisting pattern, where the $y$-component of the director remains zero, satisfies the first-order optimality conditions. However, for these Frank constants it is well known that a twist configuration incorporating a nonplanar tilt is energetically optimal $[35,46]$. Thus, there are multiple solutions satisfying (3.2)-(3.4). Furthermore, these nonplanar twist solutions only become energetically optimal for certain Frank constant ratios. For instance, such configurations are not detectable when using the one-constant approximation [13,46].

For the deflation solves, two initial guesses are constructed at each refinement level to serve as starting points for the discovery of additional solutions. Through the interior of the domain, both initial guesses are isolated to the $x y$-plane and incorporate a slight uniform tilt; see Appendix B. As discussed in [5], convergence to the energetically optimal solution can be attained even when choosing a relatively naïve initial guess. However, without deflation, the poor initial guesses used here result in convergence on all grids to a single planar twist solution, which represents only a local minimum. The first guess is also used for the initial coarse-grid, undeflated iterations.

The undeflated iterations converge to the planar twist solution displayed in Figure 4.1(a) with a final free energy of 3.701 and consume a total of 11.9 WUs as the solution is continued through the NI hierarchy. The remainder of the solutions are located 
using deflation. The configurations in Figure 4.1(b) and (c) represent the energetically optimal structures for this problem, with both exhibiting final free energies of 3.593. Newton solves for these solutions require 17.8 and $18.0 \mathrm{WUs}$, respectively. The symmetry of the device and boundary conditions allow for the reflective symmetry seen in these two solutions.

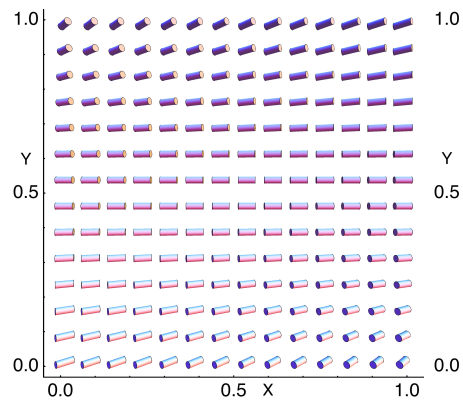

(a)

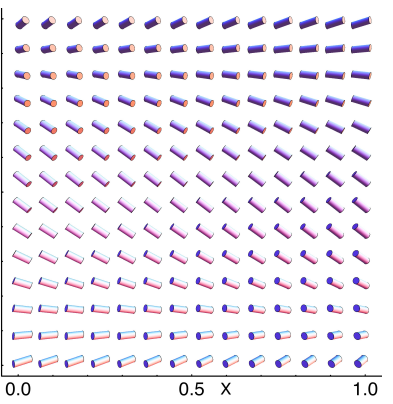

(b)

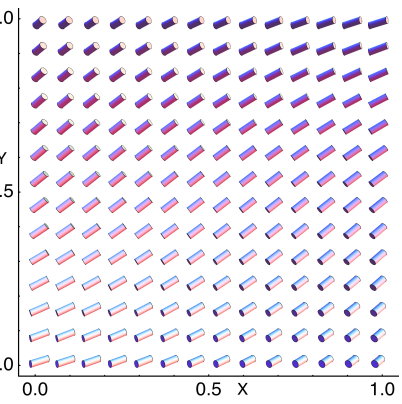

(c)

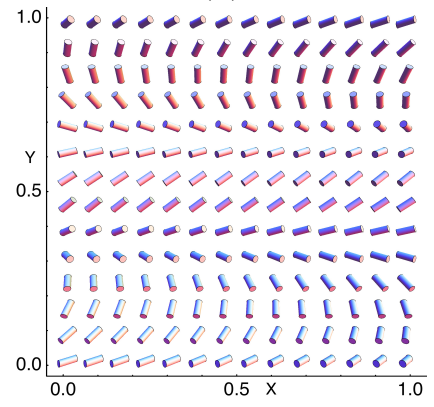

(d)

Fig. 4.1: (a) Resolved non-minimizing solution on $256 \times 256$ mesh (restricted for visualization) with final free energy of 3.701. (b) Energy-minimizing solution identified through deflation with final free energy of 3.593. (c) Symmetric energy-minimizing solution found with deflation. All solutions are located on the coarsest mesh. (d) Intricate non-minimizing solution satisfying the optimality conditions located with deflation parameters $\alpha=0.1$ and $p=2.0$ and starting with a $4 \times 4$ coarse mesh.

For comparison, simulations without NI are performed using deflation damping parameters of $\omega_{2}=1.0$ and 0.2 . In either case, standard Newton iterations use a total of $77 \mathrm{~V}$-cycles (WUs) to find the configuration in Figure 4.1(a). For $\omega_{2}=1.0$, subsequent deflation iterations fail to locate any additional distinct solutions. On the other hand, with $\omega_{2}=0.2$, both arrangements shown in Figure 4.1(b) and (c) are found but each incurs a cost of $687 \mathrm{~V}$-cycles (WUs), representing a significant increase in overall work necessary to compute these solutions. For this problem, NI clearly improves both the efficacy and efficiency of the deflation approach.

Finally, we consider the computational effects associated with adjusting the deflation parameters, $\alpha$ and $p$, summarized in Table 4.1. The value in each cell represents the total number of WUs required in computing all discovered solutions. Each combination of parameters yields the three solutions shown in Figure 4.1(a)-(c), providing experimental evidence for the robustness of the deflation approach with respect to parameter choice. However, the applied parameters do have a noticeable effect on the work unit efficiency. These fluctuations correspond to changes in the mesh level 
of the NI hierarchy at which deflation successfully locates additional solutions. For example, the accrued WUs for $\alpha=0.1$ and $p=1.0$ are considerably higher than those of $\alpha=1.0$ and $p=3.0$ because the third liquid crystal configuration is discovered on the fourth mesh rather than the coarsest one.

\begin{tabular}{|c|c|c|c|c|}
\hline$\alpha \backslash p$ & 1.0 & 2.0 & 3.0 & 4.0 \\
\hline 0.1 & 82.6 & 50.8 & 47.7 & 47.9 \\
\hline 0.5 & 65.5 & 49.3 & 66.0 & 66.0 \\
\hline 1.0 & 64.9 & 65.8 & 47.8 & 57.0 \\
\hline 2.0 & 65.5 & 56.9 & 66.0 & 57.4 \\
\hline
\end{tabular}

Table 4.1: Total WUs accumulated within the NI hierarchy during the computation of the three configurations shown in Figure 4.1(a)-(c) for varying deflation parameters. Note that these WUs do not include overhead associated with deflation iterations that did not converge on each grid.

In other experiments, certain setups and selections of deflation parameters may yield additional distinct solutions. For example, the configuration displayed in Figure $4.1(\mathrm{~d})$ is discovered when applying deflation parameters of $\alpha=0.1$ and $p=2.0$ with an NI hierarchy starting on a $4 \times 4$ coarse mesh. The structure's free energy is 32.336 . While the configuration is clearly not energetically optimal, it satisfies the first-order optimality conditions. Further, in Section 5.2, the discovery of an additional, distinct solution with the application of alternative deflation parameters without changing the NI hierarchy, is briefly discussed. At present, deflation parameter choice generally relies on numerical experience and experimentation, but current work is focused on constructing a better theoretical understanding of these parameters' effects on convergence and constructing techniques for automatic selection of these values.

4.2. Freedericksz transition. The second numerical experiment considers a classical Freedericksz transition problem with simple director boundary conditions such that $\mathbf{n}$ lies uniformly parallel to the $x$-axis at the edges $y=0$ and $y=1$. For the electric potential, $\phi$, the boundary conditions set $\phi(x, 0)=0$ and $\phi(x, 1)=V=1.1$. The relevant Frank and electric constants are $K_{1}=1, K_{2}=0.62903$, and $K_{3}=$ 1.32258 (those of 5CB), $\epsilon_{0}=1.42809, \epsilon_{\perp}=7$, and $\epsilon_{a}=11.5$. Note that for $\epsilon_{a}>0$ the liquid crystals are attracted to alignment parallel to the electric field. The relevant damping parameters are $\omega_{1}=1.0, \Delta_{1}=0.0, \omega_{2}=1.0$, and $\Delta_{2}=0.5$. The same two initial guesses for $\mathbf{n}$ used in the previous experiment are applied in the deflation solves here; c.f. Appendix B. These configurations serve as the starting point for all deflation searches in the NI hierarchy.

The initial undeflated iterations converge to the elastic rest configuration uniformly parallel to the $x$-axis shown in Figure $4.2(\mathrm{a})$ and use 16.0 WUs. The final free energy for this structure is -6.048 . Thereafter, using deflation, the energetically optimal arrangements displayed in Figures 4.2(b) and (c) are found and both have final free energies of -6.778 . The computation of each solution requires 33.4 WUs. These solutions represent a true Freedericksz transition in which the applied electric field successfully deforms the nematic configuration away from elastic rest. Without deflation, the two guesses used here converge to the same solution, Figure 4.2(a), on all grids. Alternatively, applying the deflation technique without NI for $\omega_{2}=1.0$ and 0.2 , standard Newton iterations converge to the configuration in Figure 4.2(a) using $48 \mathrm{~V}$-cycles (WUs) and subsequent deflation iterations miss the energetically optimal structures for either damping parameter. 


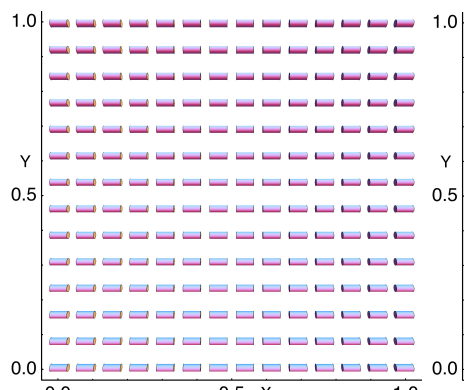

(a)

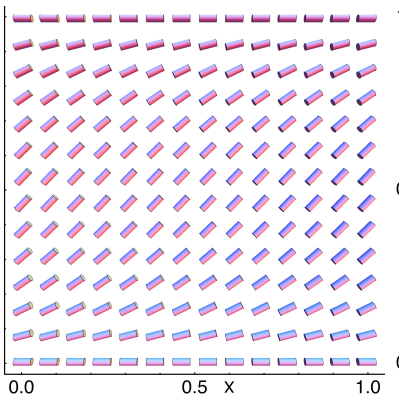

(b)

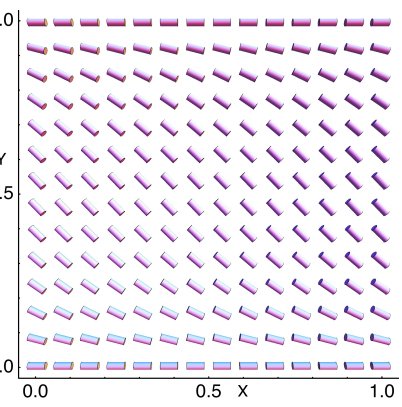

(c)

Fig. 4.2: (a) Resolved non-minimizing solution on $256 \times 256$ mesh (restricted for visualization) with final free energy of -6.048 . (b) Energy-minimizing solution found through deflation with final free energy of -6.778 . (c) Symmetric energy-minimizing solution computed with deflation. All solutions were located on the coarsest mesh.

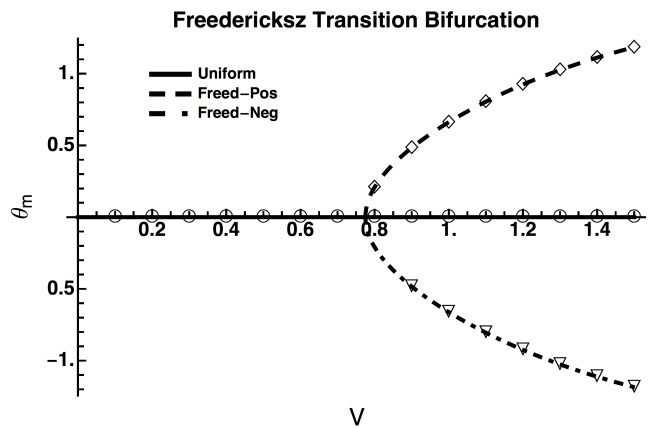

(a)

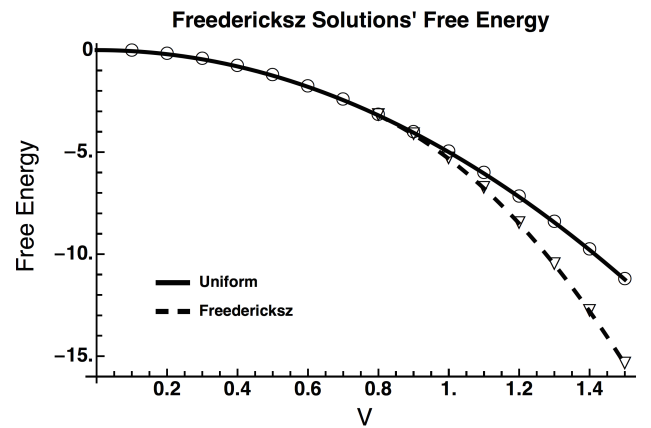

(b)

Fig. 4.3: (a) Pitchfork bifurcation diagram characterizing the Freedericksz transition at approximately $V_{c}=0.775$. Lines depict analytical values for $\theta_{m}$ while markers indicate maximum angular tilt for solutions obtained through deflation. (b) A plot of free energy as a function of applied voltage. Lines are analytical free energies and markers denote free energies for solutions obtained through deflation.

The Freedericksz transition problem exhibits a well-known and important pitchfork bifurcation. The strength of the applied voltage at the top substrate, $V$, relative to the elastic characteristics of the liquid crystal, determines this bifurcation structure. Retaining the liquid crystal constants outlined above and varying the applied voltage, we observe the bifurcation process. As the applied voltage becomes stronger, the electric field begins to overpower the elastic effects in the sample. At a critical threshold, given analytically by $V_{c}=\pi \sqrt{\frac{K_{1}}{\epsilon_{0} \epsilon_{a}}}$, it becomes energetically advantageous to tilt in the direction of the field $[46,49]$. The critical voltage for the problem parameters considered here is $V_{c}=0.775$.

In Figure 4.3(a), when $V$ reaches the critical value, solutions tilting in the direction of the electric field begin to satisfy the first-order optimality conditions and yield optimal free energy. The value $\theta_{m}$ denotes the maximum angular tilt of the director field in the direction of the electric field resulting from the applied voltage. Figure 4.3(b) characterizes the shift in free-energy optimizing solutions resulting from the Freedericksz transition as $V$ passes the critical voltage, $V_{c}$. In both figures, the lines 
represent analytical computations as $V$ varies [46], and the individual markers are values for solutions computed independently for each value of $V$ through deflation.

4.3. Escape and disclination solutions. This third numerical experiment investigates the phenomenon of defects, also known as disclinations. Defects in liquid crystal structures are locations in a sample where the director field is undefined or contains discontinuities. There are a multitude of disclination types including point, wedge, sheet, and loop defects, among others. In this example, we consider wedge disclinations. These disclinations involve rotation around an axis parallel to the defect and are, therefore, sometimes referred to as axial disclinations [25]. Wedge-type disclinations have been studied in $[19,23]$.

For this simulation, the damping parameters are $\omega_{1}=0.4, \Delta_{1}=0.2, \omega_{2}=$ 1.0, and $\Delta_{2}=0.5$. Dirichlet boundary conditions are applied to the entire domain boundary and no electric field is present. The boundary conditions are fixed such that the director faces the center of the domain and Frank constants of $K_{1}=1.0$, $K_{2}=3.0$, and $K_{3}=1.2$ are used. As in the previous experiments, two initial guesses, detailed in Appendix B, are used for the deflation solves on each grid.

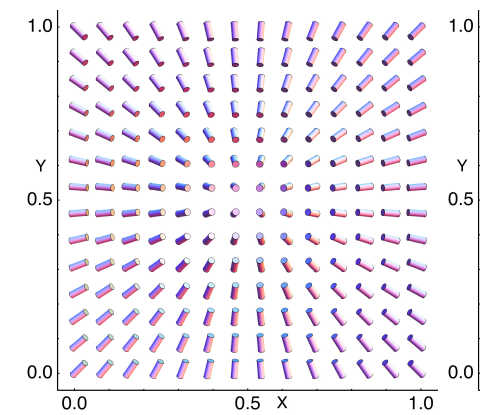

(a)

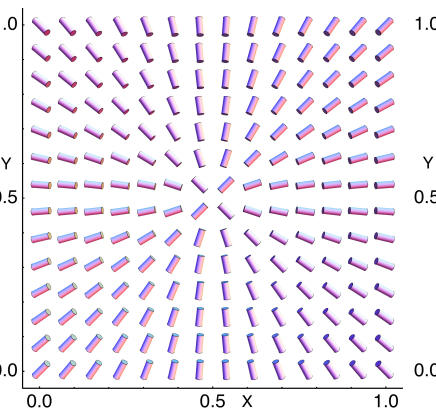

(b)

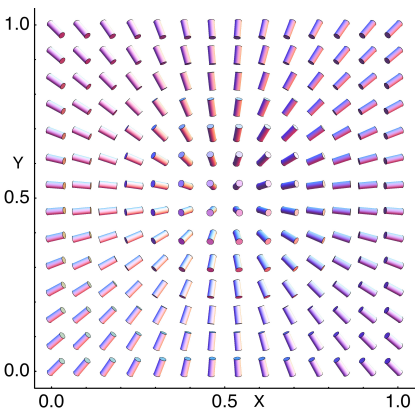

(c)

Fig. 4.4: (a) Resolved escape solution on $256 \times 256$ mesh (restricted for visualization) with final free energy of 9.971. (b) Disclination solution with central wedge defect and final free energy of 24.042 (free energy is expected to diverge with refinement). (c) Symmetric escape solution with final free energy of 9.971 .

\begin{tabular}{|c|c|c|c|c|c|c|}
\hline Grid & $8 \times 8$ & $16 \times 16$ & $32 \times 32$ & $64 \times 64$ & $128 \times 128$ & $256 \times 256$ \\
\hline Pos. Escape & 9.972 & 9.971 & 9.971 & 9.971 & 9.971 & 9.971 \\
\hline Disclination & 13.154 & 15.331 & 17.509 & 19.686 & 21.864 & 24.042 \\
\hline Neg. Escape & 9.971 & 9.971 & 9.971 & 9.971 & 9.971 & 9.971 \\
\hline
\end{tabular}

Table 4.2: Computed free energies on each mesh for the set of computed solutions.

The first solution, located using undeflated solves, is displayed in Figure 4.4(a). This director field is continuous and shares some similarities with the solutions found in $[11,39]$ for long cylindrical capillaries. The progression of the solves consumes 38.4 WUs. The solution displayed in Figure 4.4(c) is a second, symmetric configuration computed in the deflation solves using 39.5 WUs. The calculated free energy on each mesh for both solutions is shown in Table 4.2. Due to the symmetric composition of the device, zenithal tilt in either direction results in an optimal arrangement.

In Figure 4.4(b), the remaining solution generated through deflation using 121.0 WUs is displayed. Without deflation, only two of these three solutions would be 
found across all NI grids. This configuration reveals a disclination where the director field becomes undefined at the center of the domain. The existence of this type of solution lends credence to the escape solution moniker given to configurations like those in Figures 4.4(a) and 4.4(c), as the director "escapes" in the $z$-direction to avoid the defect. The disclination structure does not have finite free energy, as the functional values diverge as they approach the central defect [46]. Since the solution is approximated with finite elements, this divergent behavior is manifest in a monotonically increasing free energy after each successive refinement; see Table 4.2. The free energy of 24.042 computed on the finest grid is expected to continue to rise as the domain is more finely discretized. These types of disclinations can be synthesized and observed under certain conditions [45].

The distribution of nonlinear iterations across the NI levels for the escape and disclination configurations is shown in Table 4.3. The iteration totals associated with a particular solution are those Newton steps that converged to that solution on the given mesh. The counts marked in bold are iterations performed as part of the discovery stage of the algorithm using deflation. The total "anonymous" iteration counts in the last column are those deflation steps that resulted in divergence from the unit-length constraint or reached the Newton iteration limit without converging. The size of this iteration overhead depends on the number of initial guesses used and the complexity of the configurations. While the configurations in this simulation are found on the first grid, using different deflation parameters can change this outcome. For instance, using $\alpha=0.1$ and $p=1.0$, the solutions in Figures 4.4(b) and (c) are not discovered until the $64 \times 64$ grid.

\begin{tabular}{|c||c|c|c|c|}
\hline \multicolumn{1}{|c||}{} & \multicolumn{4}{c|}{ Figure 4.4 } \\
\hline Grid & (a) & (b) & (c) & Total Anon. \\
\hline $8 \times 8$ & 23 & $\mathbf{7}$ & $\mathbf{1 0 0}$ & - \\
\hline $16 \times 16$ & 9 & 12 & 9 & $\mathbf{1 0 2}$ \\
\hline $32 \times 32$ & 5 & 8 & 5 & $\mathbf{2 0 0}$ \\
\hline $64 \times 64$ & 2 & 5 & 2 & $\mathbf{2 0 0}$ \\
\hline $128 \times 128$ & 2 & 5 & 2 & $\mathbf{2 0 0}$ \\
\hline $256 \times 256$ & 2 & 5 & 2 & $\mathbf{2 0 0}$ \\
\hline
\end{tabular}

Table 4.3: Newton iteration counts across grids directly attributable to a solution along with those resulting in divergence or tolerance stoppage in the deflation process for the disclination problem. Bold numbers are associated with the discovery stage using deflation.

In considering the influence of NI on the efficacy of the deflation technique for this problem, two simulations without NI are undertaken that employ deflation damping values of $\omega_{2}=1.0$ and 0.2 , respectively. In both experiments the arrangement shown in Figure 4.4(a) is found with standard Newton iterations using a total of $359 \mathrm{~V}$ cycles (WUs). Using the first deflation damping value, both sets of deflated Newton iterations diverge, failing to locate additional solutions. This result is improved by applying the second, smaller damping parameter, for which the solution in Figure 4.4(c) is discovered consuming $775 \mathrm{~V}$-cycles (WUs). However, in either case, the disclination solution remains undiscovered. A possible cause for missing this solution is the fact that the coarse-grid representations of the escape and disclination solutions are energetically closer to one another compared with those of finer-scale meshes, as seen in Table 4.2. As the disclination configuration's free energy diverges, the likelihood of deflated iterations reaching its basin of attraction may shrink. 


\begin{tabular}{|c||c|c|c||c|c|c||c|c|c|}
\hline \multicolumn{1}{|c||}{} & \multicolumn{3}{c||}{ Fig. 4.1: Tilt-Twist } & \multicolumn{3}{c||}{ Fig. 4.2: Freeder. } & \multicolumn{3}{c|}{ Fig. 4.4: Disclination } \\
\hline Grid & (a) & (b) & (c) & (a) & (b) & (c) & (a) & (b) & (c) \\
\hline $8 \times 8$ & 10.8 & $\mathbf{1 1 . 4}$ & $\mathbf{1 2 . 1}$ & 10.7 & $\mathbf{1 0 . 1}$ & $\mathbf{1 0 . 1}$ & 12.9 & $\mathbf{1 3 . 3}$ & $\mathbf{1 3 . 9}$ \\
\hline $16 \times 16$ & 8.0 & 10.5 & 10.5 & 10.7 & 11.0 & 11.0 & 13.0 & 14.9 & 13.0 \\
\hline $32 \times 32$ & 8.0 & 11.5 & 11.5 & 11.0 & 12.0 & 12.0 & 13.0 & 14.2 & 13.0 \\
\hline $64 \times 64$ & 8.5 & 12.0 & 12.0 & 12.0 & 12.5 & 12.5 & 13.5 & 16.6 & 13.5 \\
\hline $128 \times 128$ & 9.0 & 11.5 & 11.5 & 12.0 & 12.5 & 12.5 & 14.0 & 17.8 & 14.0 \\
\hline $256 \times 256$ & 6.0 & 10.0 & 10.0 & 12.0 & 12.5 & 12.5 & 14.0 & 18.2 & 14.0 \\
\hline
\end{tabular}

Table 4.4: Average multigrid iteration counts on each mesh during progression of the NI hierarchy for solutions from the experiments in Section 4. Counts in bold represent average iterations for linear solves on deflated systems.

Finally, the performance of the linear solver for the three experiments above is illustrated in Table 4.4. The table displays multigrid iteration counts averaged over Newton steps on each mesh for the solutions found in each experiment. Bold values delineate average iterations for deflated linear systems. These iteration counts are relatively small and remain steady across mesh refinements. Note that the iteration counts associated with the deflated linear solves are consistent with the performance of the solver on the undeflated systems. These results are especially promising as no special modifications to the solver are necessary for integration with the deflation technique.

5. Cholesteric liquid crystals. In our final experiment, we consider cholesteric liquid crystals, which share many properties with nematics but have slightly less symmetry due to chirality. In particular, their inherent helical structure leads to a property known as enantiomorphy where cholesteric molecules are distinguishable from their reflected images. Right-handed helical cholesteric structures are transformed to left-handed helixes upon reflection. This asymmetry leads to a modest modification of the elastic free-energy functional for these types of liquid crystals and a fourth (nondimensionalized) physical constant, $t_{0}$, which characterizes the chiral properties of the cholesteric liquid crystal and may be positive or negative depending on the handedness of the cholesteric [15]. The full free-energy functional, ignoring electric effects, is written

$$
\begin{aligned}
\mathcal{C}_{0}(\mathbf{n})= & K_{1}\langle\nabla \cdot \mathbf{n}, \nabla \cdot \mathbf{n}\rangle_{0}+K_{2}\left\langle\mathbf{n} \cdot \nabla \times \mathbf{n}+t_{0}, \mathbf{n} \cdot \nabla \times \mathbf{n}+t_{0}\right\rangle_{0} \\
& +K_{3}\langle\mathbf{n} \times \nabla \times \mathbf{n}, \mathbf{n} \times \nabla \times \mathbf{n}\rangle_{0} \\
= & \mathcal{F}(\mathbf{n}, 0)+2 K_{2}\left\langle t_{0}, \mathbf{n} \cdot \nabla \times \mathbf{n}\right\rangle_{0}+K_{2}\left\langle t_{0}, t_{0}\right\rangle_{0},
\end{aligned}
$$

where $\mathcal{F}(\mathbf{n}, 0)$ is the nematic functional in (3.1). Note that the last term does not depend on $\mathbf{n}$. Thus, in the minimization process, we need not include that term. Hence, we define the cholesteric free-energy functional to be minimized as

$$
\mathcal{C}(\mathbf{n})=\mathcal{F}(\mathbf{n}, 0)+2 K_{2}\left\langle t_{0}, \mathbf{n} \cdot \nabla \times \mathbf{n}\right\rangle_{0} .
$$

5.1. Minimization. Since cholesterics are subject to the same pointwise unitlength constraint as nematics, the Lagrangian is formed as

$$
\mathcal{L}^{C}(\mathbf{n}, \lambda)=\mathcal{C}(\mathbf{n})+\int_{\Omega} \lambda(\mathbf{n} \cdot \mathbf{n}-1) d V .
$$


Computing the derivative of $\mathcal{L}^{C}$ with respect to $\mathbf{n}$ yields

$$
\mathcal{L}_{\mathbf{n}}^{C}(\mathbf{n}, \lambda)[\mathbf{w}]=\mathcal{L}_{\mathbf{n}}[\mathbf{w}]+2 K_{2}\left(\left\langle t_{0}, \mathbf{w} \cdot \nabla \times \mathbf{n}\right\rangle_{0}+\left\langle t_{0}, \mathbf{n} \cdot \nabla \times \mathbf{w}\right\rangle_{0}\right) .
$$

Because the additional terms of the free energy specific to cholesterics do not depend on $\lambda$, derivatives of this Lagrangian involving $\lambda$ are identical to the nematic case. Thus, in computing the Hessian, the only derivative with additional terms is the second-order derivative with respect to $\mathbf{n}$, giving

$$
\mathcal{L}_{\mathbf{n n}}^{C}=\mathcal{L}_{\mathbf{n n}}+2 K_{2}\left(\left\langle t_{0}, \mathbf{w} \cdot \nabla \times \delta \mathbf{n}\right\rangle_{0}+\left\langle t_{0}, \delta \mathbf{n} \cdot \nabla \times \mathbf{w}\right\rangle_{0}\right) .
$$

Modifying the energy-minimization and deflation algorithm discussed above for nematics by adding in the appropriate terms corresponding to the cholesteric free energy yields an effective algorithm for computing multiple equilibrium configurations of cholesteric liquid crystals.

5.2. Chiral configuration. In this simulation, we consider a simple cholesteric configuration, using the same mixed periodic and Dirichlet boundary conditions and slab domain assumption as in previous numerical examples. At the Dirichlet boundary, uniform conditions such that $\mathbf{n}=(1,0,0)$ are enforced. In the case of nematic liquid crystals, subject to elastic forces, the minimizing configuration is full alignment parallel to the director on the boundary. However, the energetically optimal arrangement for cholesterics is a chiral configuration along the $y$-axis with twist properties determined by the value of $t_{0}$. Using an ansatz for a chiral solution of the form $\mathbf{n}=(\cos (\tau y), 0,-\sin (\tau y))$, the computations in [46] can be modified to our coordinate system, giving the elastic free energy associated with this ansatz as $\frac{1}{2} K_{2}\left(t_{0}-\tau\right)^{2}|\Omega|$, where $|\Omega|$ is the domain measure, so long as the chiral ansatz also conforms to the imposed boundary conditions. Since the elastic free energy is positive and semi-definite, clearly the free energy of the ansatz is minimized when $\tau=t_{0}$; when $t_{0}$ is an integer multiple of $2 \pi$, the uniform Dirichlet boundary conditions above will also be satisfied.

For this numerical simulation, the Frank constants are set to $K_{1}=1.0, K_{2}=3.0$, and $K_{3}=1.2$, while $t_{0}=-2 \pi$. This implies that the energy-minimizing solution corresponds to a left-handed helix running parallel to the $y$-axis with a $2 \pi$-rotation across the device. However, additional configurations, while not globally minimizing, satisfy the first-order optimality conditions and are experimentally observable.

The deflation algorithm is applied with damping values of $\omega_{1}=0.2, \Delta_{1}=0.2$, $\omega_{2}=0.2$, and $\Delta_{2}=0.0$. Using the set of three initial guesses outlined in Appendix $\mathrm{B}$, the algorithm reveals a rich set of solutions satisfying the optimality conditions. A total of six distinct solutions, shown in Figure 5.1, are found, whereas, without deflation, only three solutions would be identified across all grids. The corresponding, computed free energies for these solutions is shown in Table 5.1 along with average iteration counts for the multigrid-preconditioned GMRES linear solver. In general, the linear solver iteration counts are higher for these cholesteric systems compared with those of the previous section. Correspondingly, the WUs, shown in the same table, are larger when compared with previous experiments. This increase in iterations is most likely due to a combination of the additional term in the cholesteric functional and higher overall free energies in the solutions. However, the iterations counts are relatively consistent across grid refinements, and the average solver iterations for the deflated systems, shown in bold, correspond well with the iteration counts for the undeflated solves.

The solution set includes degenerate planar solutions displayed in Figures 5.1(a) and (d). By virtue of the chirality of cholesterics, these configurations are not globally 


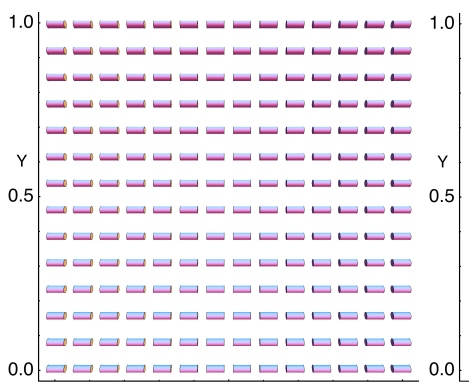

(a)

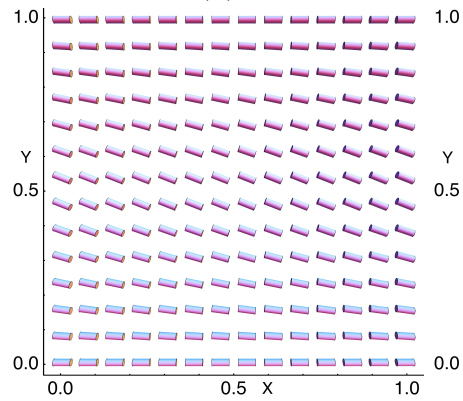

(d)

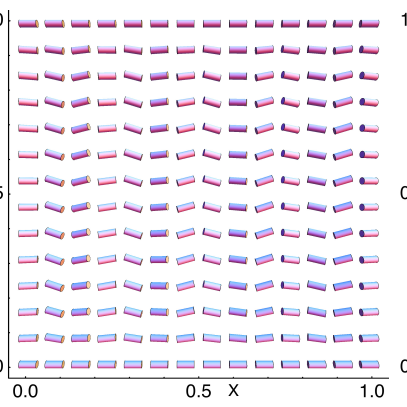

(b)

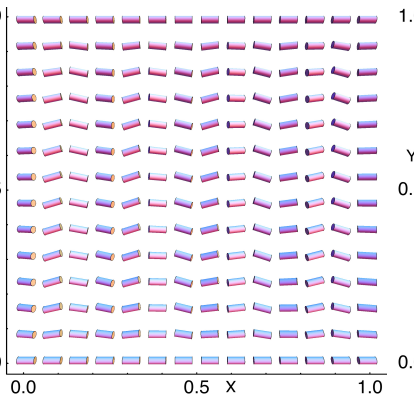

(e)

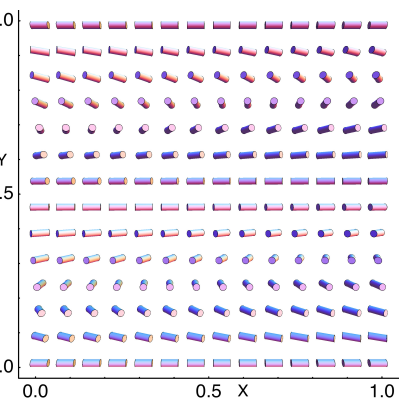

(c)

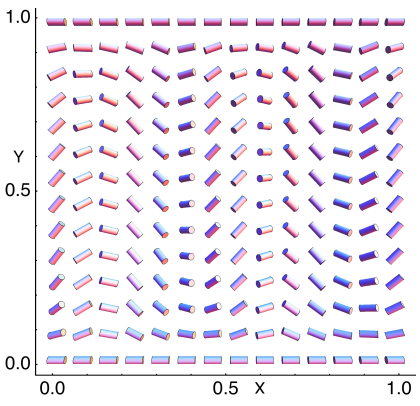

(f)

Fig. 5.1: Family of distinct solutions for the cholesteric equilibrium problem found through deflation. Each solution is computed computed on a $256 \times 256$ mesh and restricted for visualization. The energy minimizing solution is displayed in (c).

\begin{tabular}{|c||c|c|c|c|c|c|}
\hline \multicolumn{1}{|c||}{} & \multicolumn{7}{c|}{ Figure 5.1} \\
\hline Grid & (a) & (b) & $($ c) & $($ d) & $(\mathrm{e})$ & $(\mathrm{f})$ \\
\hline $8 \times 8$ & 46.2 & $\mathbf{5 2 . 9}$ & $\mathbf{1 1 . 3}$ & - & - & - \\
\hline $16 \times 16$ & 66.0 & 54.3 & 9.0 & $\mathbf{6 7 . 7}$ & $\mathbf{6 6 . 8}$ & - \\
\hline $32 \times 32$ & 65.0 & 33.2 & 8.0 & 53.9 & 34.1 & - \\
\hline $64 \times 64$ & 61.0 & 28.4 & 8.0 & 35.8 & 26.9 & - \\
\hline $128 \times 128$ & 62.0 & 33.0 & 9.0 & 52.5 & 32.5 & $\mathbf{2 9 . 0}$ \\
\hline $256 \times 256$ & 78.0 & 30.5 & 9.5 & 46.0 & 30.0 & 18.5 \\
\hline Work Units & 100.7 & 103.7 & 28.5 & 156.9 & 108.7 & 493.0 \\
\hline Free Energy & 59.218 & 56.553 & $2.984 \mathrm{e}-08$ & 59.378 & 56.553 & 31.821 \\
\hline
\end{tabular}

Table 5.1: Average multigrid iteration counts on each mesh during progression of the NI hierarchy for the cholesteric experiment above. Counts in bold represent average iterations for linear solves on deflated systems. The final rows display the WUs and free energy associated with each computed equilibrium configuration.

minimizing. Also included in the computed arrangements are structures with left-hand twists following the $x$-axis. These behaviors decrease each system's free energy below that of the planar solutions. For instance, the free energy of the configuration in Figure 5.1(f) is 31.821, well below the free energy of 59.218 for the solution in Figure 5.1(a). Similar transverse wave-like structures are observable in certain cholesteric samples. Note that this solution is not found until the fifth mesh in the NI framework. Furthermore, if the deflation parameters are adjusted, a seventh configuration of this 
type, not shown here, is also located during the deflation process.

The arrangement displayed in Figure 5.1(c), is energetically optimal. As suggested in the analysis above, the director profile contains a left-handed helical structure rotating $2 \pi$ radians about an axis parallel to the $y$-axis and has a computed free energy of 2.984e-08. It should be noted that in order to obtain this solution, an initial guess incorporating a twisting profile is used. Moving in the configuration space from a profile with little or no twist to one that incorporates a full $2 \pi$-rotation is far from monotonic in terms of energy optimization. For example, introducing a moderate twist into the planar solutions of Figures 5.1(a) or (d) increases their free energy until the twist approaches a $2 \pi$-rotation. The minima valleys are well-separated and the strength of the poles introduced through deflation are often not enough to overcome the barrier dividing the valleys. Investigation into the application of generalized tunneling methods, which have been used to address some aspects of this challenge in the context of function minimization [36], will be the subject of future work.

As in Table 4.3, the nonlinear iterations accrued on each mesh during the NI process are presented in Table 5.2. Iteration counts in bold delineate those performed using deflation. The deflation iteration counts are generally higher with the increased Newton damping in the deflation stage. Further, distinct solutions are discovered through deflation on multiple grids. This is especially apparent in the discovery of the structure in Figure 5.1(f), which is only found on the second finest mesh. Thus, eliminating or reducing deflation iterations on finer grids is difficult without risking the loss of additional solutions.

\begin{tabular}{|c||c|c|c|c|c|c|c|}
\hline \multicolumn{1}{|c||}{} & \multicolumn{9}{c|}{ Figure 5.1 } \\
\hline Grid & (a) & (b) & (c) & (d) & (e) & (f) & Total Anon. \\
\hline $8 \times 8$ & 46 & $\mathbf{5 6}$ & $\mathbf{5 0}$ & - & - & - & $\mathbf{1 0 0}$ \\
\hline $16 \times 16$ & 1 & 22 & 19 & $\mathbf{8 7}$ & $\mathbf{5 5}$ & - & $\mathbf{1 0 0}$ \\
\hline $32 \times 32$ & 1 & 12 & 10 & 8 & 12 & - & $\mathbf{2 2 8}$ \\
\hline $64 \times 64$ & 1 & 7 & 5 & 4 & 7 & - & $\mathbf{2 3 3}$ \\
\hline $128 \times 128$ & 1 & 2 & 2 & 2 & 2 & $\mathbf{6 3}$ & $\mathbf{2 0 0}$ \\
\hline $256 \times 256$ & 1 & 2 & 2 & 2 & 2 & 2 & $\mathbf{2 5 3}$ \\
\hline
\end{tabular}

Table 5.2: Newton iteration counts across grids directly attributable to a solution along with those resulting in divergence or tolerance stoppage in the deflation process for the cholesteric problem. Bold numbers are associated with the discovery stage using deflation.

Finally, without NI, only the arrangements of Figures 5.1(a), (c), and (f) are found with 2344, 674, and $1847 \mathrm{~V}$-cycles (WUs), respectively. In addition to the loss of at least three solutions, these WU totals indicate that the combination of NI and deflation reveals six solutions with comparable work to computing any single solution for deflation without NI.

6. Conclusions and future work. In this paper, a combined deflation-nested iteration strategy for efficiently computing multiple solutions to nonlinear variational systems was presented. It is demonstrated to have significant advantages over applying the existing deflation methodology on a single grid. In particular, the combined approach retains the efficiency expected of NI algorithms for nonlinear PDEs while enhancing the deflation approach's ability to expose multiple solutions. As with the original deflation methodology, we are able to reuse existing linear solvers for linearizations of the undeflated system, further enhancing the computational efficiency 
of the combined approach.

Four numerical simulations were conducted with the combined algorithm, demonstrating the effectiveness of this approach for problems in the numerical simulation of nematic and cholesteric liquid crystals. In all cases, multiple solutions are found, including global minima, and correct bifurcation structures are computed for the freeenergy model in two cases where these are known analytically. The effectiveness of the algorithm considered here is expected to inform the application of the deflation method for a more general class of constrained optimization and multiphysics problems, including closely related models in ferromagnetics. Future work will consider construction of a generalized tunneling approach, based on the work in [36], applied to the Newton iterations to further increase the power of the deflation method. In addition, we aim to investigate the method's performance in analyzing new physical phenomena and behaviors in shaped domains. Finally, strategies for adaptive construction of initial guesses for the deflation method at each level of the NI process will be studied.

Acknowledgments. The authors would like to thank Professor Timothy Atherton for his useful suggestions and guidance and Dr. Thomas Benson for allowing us to adapt his code. We would also like to thank the anonymous referees for their helpful comments that improved the quality of this manuscript.

\section{REFERENCES}

[1] J. H. Adler, T. J. Atherton, T. R. Benson, D. B. Emerson, and S. P. Maclachlan, Energy minimization for liquid crystal equilibrium with electric and flexoelectric effects, SIAM J. Sci. Comput., 37 (2015), pp. S157-S176.

[2] J. H. Adler, T. J. Atherton, D. B. Emerson, and S. P. Maclachlan, An energyminimization finite-element approach for the Frank-Oseen model of nematic liquid crystals, SIAM J. Numer. Anal., 53 (2015), pp. 2226-2254.

[3] J. H. Adler, T. R. Benson, E. C. Cyr, S. P. Maclachlan, and R. S. Tuminaro, Monolithic multigrid methods for two-dimensional resistive magnetohydrodynamics, SIAM J. Sci. Comput., 38 (2016), pp. B1-B24.

[4] J. H. Adler, J. Brannick, C. Liu, T. Manteuffel, and L. Zikatanov, First-order system least squares and the energetic variational approach for two-phase flow, J. Comput. Phys., 230 (2011), pp. 6647-6663.

[5] J. H. Adler, D. B. Emerson, S. P. Maclachlan, and T. A. Manteuffel, Constrained optimization for liquid crystal equilibria, SIAM J. Sci. Comput., 38 (2016), pp. B50-B76.

[6] J. H. Adler, T. A. Manteuffel, S. F. McCormick, J. W. Ruge, and G. D. Sanders, Nested iteration and first-order system least squares for incompressible, resistive magnetohydrodynamics, SIAM J. Sci. Comput., 32 (2010), pp. 1506-1526.

[7] T. J. Atherton And J. H. Adler, Competition of elasticity and flexoelectricity for bistable alignment of nematic liquid crystals on patterned surfaces, Phys. Rev. E, 86 (2012).

[8] T. J. Atherton and J. R. Sambles, Orientational transition in a nematic liquid crystal at a patterned surface, Phys. Rev. E, 74 (2006).

[9] W. Bangerth, R. Hartmann, and G. Kanschat, deal.II - a general purpose object oriented finite element library, ACM Trans. Math. Softw., 33 (2007), pp. 24/1-24/27.

[10] R. H. Byrd, R. B. Schnabel, and G. A. Shultz, A trust region algorithm for nonlinearly constrained optimization, SIAM J. Numer. Anal., 24 (1987), pp. 1152-1170.

[11] P. E. Cladis And M. KlÉman, Non-singular disclinations of strength $S=+1$ in nematics, J. de Physique, 33 (1972), pp. 591-598.

[12] A. L. Codd, T. A. Manteuffel, And S. F. MCConmick, Multilevel first-order system least squares for nonlinear elliptic partial differential equations, SIAM J. Numer. Anal., 41 (2003), pp. 2197-2209.

[13] R. Cohen, R. Hardt, D. Kinderlehrer, S. Lin, and M. Luskin, Minimum energy configurations for liquid crystals: Computational results, in Theory and Applications of Liquid Crystals, vol. 5 of The IMA Volumes in Mathematics and Its Applications, Springer-Verlag, New York, 1987, pp. 99-121. 
[14] P. J. Collings, Liquid Crystals: Nature's Delicate Phase of Matter, Bristol, 1990.

[15] P. J. Collings And M. Hird, Introduction to Liquid Crystals, Taylor and Francis, London, UK, 1997.

[16] T. A. Davis and E. C. Gartland-Jr., Finite element analysis of the Landau-de Gennes minimization problem for liquid crystals, SIAM J. Numer. Anal., 35 (1998), pp. 336-362.

[17] P. G. De Gennes and J. Prost, The Physics of Liquid Crystals, Clarendon Press, Oxford, UK, 2nd ed., 1993.

[18] H. J. Deuling, Deformation of nematic liquid crystals in an electric field, Mol. Cryst. Liq. Cryst., 19 (1972), pp. 123-131.

[19] I. E. DzylaoshinskiI, Theory of disclinations in liquid crystals, Sov. Phys. JETP, 31 (1970), pp. $773-777$.

[20] J. L. ERICKSEn, Hydrostatic theory of liquid crystals, Arch. Rat. Mech. Anal., 9 (1962), pp. 371378.

[21] - Inequalities in liquid crystal theory, Phys. Fluids, 9 (1966), pp. 1205-1207.

[22] P. E. Farrell, Á. Birkisson, and S. W. Funke, Deflation techniques for finding distinct solutions of nonlinear partial differential equations, SIAM J. Sci. Comput., 37 (2015), pp. A2026-A2045.

[23] F. C. Frank, On the theory of liquid crystals, Discuss. Faraday Soc., 25 (1958), pp. 19-28.

[24] V. Freedericksz and V. Zolina, Forces causing the orientation of an anisotropic liquid, Trans. Faraday Soc., 29 (1933), pp. 919-930.

[25] J. Friedel And P. G. De Gennes, Boucles de disclination dans les cristaux liquides, C. R. Acad. Sc. Paris B, 268 (1969), pp. 257-259.

[26] E. C. Gartland-Jr. And A. Ramage, A renormalized Newton method for liquid crystal director models with pointwise unit-vector constraints, SIAM J. Numer. Anal., 53 (2015), pp. 251-278.

[27] L. Gattermann and A. Ritschke, Über azoxyphenoläther, Ber. Deutsche. Chem. Ges., 23 (1890), pp. 1738-1750.

[28] V. Girault and P. Raviart, Finite Element Methods for Navier-Stokes Equations: Theory and Algorithms, Springer-Verlag, Germany, 1986.

[29] M. D. Gunzburger, A. J. Meir, and J. S. Peterson, On the existence, uniqueness, and finite element approximation of solutions of the equations of stationary, incompressible magnetohydrodynamics, Math. Comp., 56 (1991), pp. 523-563.

[30] W. HAGER, Updating the inverse of a matrix, SIAM Rev., 31 (1989), pp. 221-239.

[31] Q. HU, X.-C. TAI, AND R. Winther, A saddle point approach to the computation of harmonic maps, SIAM J. Numer. Anal., 47 (2009), pp. 1500-1523.

[32] M. Kruží́ And A. Prohl, Recent developments in the modeling, analysis, and numerics of ferromagnetism, SIAM Rev., 48 (2006), pp. 439-483.

[33] J. P. F. Lagerwall and G. Scalia, A new era for liquid crystal research: Applications of liquid crystals in soft matter, nano-, bio- and microtechnology, Curr. Appl. Phys., 12 (2012), pp. 1387-1412.

[34] B. W. LeE AND N. A. ClaRK, Alignment of liquid crystals with patterned isotropic surfaces, Science, 291 (2001), pp. 2576-2580.

[35] F. M. Leslie, Distorted twisted orientation patterns in nematic liquid crystals, Pramana, Suppl. No., 1 (1975), pp. 41-55.

[36] A. V. Levy And S. Gomez, The tunneling method applied to global optimization, in Numerical Optimization, P. T. Boggs, R. H. Byrd, and R. B. Schnabel, eds., SIAM, 1985, pp. 213-244.

[37] T. A. Manteuffel, S. F. McCormick, J. G. Schmidt, and C. R. Westphal, First-order system least squares for geometrically nonlinear elasticity, SIAM J. Numer. Anal., 44 (2006), pp. 2057-2081.

[38] R. MARTí, Multi-start methods, in Handbook in Metaheuristics, F. Glover and G. A. Kochenberger, eds., vol. 57 of International Series in Operations Research \& Management Science, Springer, 2003, pp. 355-368.

[39] R. B. Meyer, On the existence of even indexed disclinations in nematic liquid crystals, Phil. Mag., 27 (1973), pp. 405-424.

[40] L. Onsager, The effects of shape on the interaction of colloidal particles, Ann. NY Acad. Sci., 51 (1949), pp. 627-659.

[41] A. Pandolfi and G. NAPOli, A numerical investigation of configurational distortions in nematic liquid crystals, J. Nonlinear Sci., 21 (2011), pp. 785-809.

[42] A. Ramage And E. C. Gartland-Jr., A preconditioned nullspace method for liquid crystal director modeling, SIAM J. Sci. Comput., 35 (2013), pp. B226-B247.

[43] R. R. Shah And N. L. Аввотt, Principles for measurement of chemical exposure based on recognition-driven anchoring transitions in liquid crystals, Science, 293 (2001), pp. 1296- 
1299.

[44] G. StARKe, Gauss-Newton multilevel methods for least-squares finite element computations of variably saturated subsurface flow, Computing, 64 (2000), pp. 323-338.

[45] M. J. Stephen and J. P. Straley, Physics of liquid crystals, Rev. Modern Phys., 46 (1974), pp. 617-704.

[46] I. W. Stewart, The Static and Dynamic Continuum Theory of Liquid Crystals: A Mathematical Introduction, Taylor and Francis, London, 2004.

[47] E. G. VirgA, Variational Theories for Liquid Crystals, Chapman and Hall, London, 1994.

[48] M. Yamada, M. Kondo, J. Mamiya, Y. Yu, M. Kinoshita, C. Barrett, and T. Ikeda, Photomobile polymer materials: Towards light-driven plastic motors, Angew. Chem. Int., 47 (2008), pp. 4986-4988.

[49] H. ZOCHER, Über die Einwirkung magnetischer, elektrischer und mechanischer Kräfte auf Mesophasen, Physik. Zietschr., 28 (1927), pp. 790-796.

\section{Appendix A. Hessian of the nematic model.}

In this appendix, we detail the full expressions for each component of the Hessian in Equation (3.5). The multiplicative notation indicates the direction associated with each derivative. For instance, $\mathcal{L}_{\lambda \mathbf{n}}[\gamma] \cdot \delta \mathbf{n}=\frac{\partial}{\partial \mathbf{n}}\left(\mathcal{L}_{\lambda}\left(\mathbf{n}_{k}, \phi_{k}, \lambda_{k}\right)[\gamma]\right)[\delta \mathbf{n}]$, where the partials indicate Gâteaux derivatives in the respective variables. Thus, each term of the Hessian is written as

$$
\begin{aligned}
& \mathcal{L}_{\mathbf{n n}}[\mathbf{w}] \cdot \delta \mathbf{n}=2 K_{1}\langle\nabla \cdot \delta \mathbf{n}, \nabla \cdot \mathbf{w}\rangle_{0}+2 K_{3}\left\langle\mathbf{Z}\left(\mathbf{n}_{k}\right) \nabla \times \delta \mathbf{n}, \nabla \times \mathbf{w}\right\rangle_{0} \\
&+2\left(K_{2}-K_{3}\right)\left(\left\langle\delta \mathbf{n} \cdot \nabla \times \mathbf{w}, \mathbf{n}_{k} \cdot \nabla \times \mathbf{n}_{k}\right\rangle_{0}\right. \\
&+\left\langle\mathbf{n}_{k} \cdot \nabla \times \mathbf{w}, \delta \mathbf{n} \cdot \nabla \times \mathbf{n}_{k}\right\rangle_{0}+\left\langle\mathbf{n}_{k} \cdot \nabla \times \mathbf{n}_{k}, \mathbf{w} \cdot \nabla \times \delta \mathbf{n}\right\rangle_{0} \\
&\left.+\left\langle\mathbf{n}_{k} \cdot \nabla \times \delta \mathbf{n}, \mathbf{w} \cdot \nabla \times \mathbf{n}_{k}\right\rangle_{0}+\left\langle\delta \mathbf{n} \cdot \nabla \times \mathbf{n}_{k}, \mathbf{w} \cdot \nabla \times \mathbf{n}_{k}\right\rangle_{0}\right) \\
& \quad-2 \epsilon_{0} \epsilon_{a}\left\langle\delta \mathbf{n} \cdot \nabla \phi_{k}, \mathbf{w} \cdot \nabla \phi_{k}\right\rangle_{0}+2 \int_{\Omega} \lambda_{k}(\delta \mathbf{n}, \mathbf{w}) d V, \\
& \mathcal{L}_{\mathbf{n} \phi}[\mathbf{w}] \cdot \delta \phi=- 2 \epsilon_{0} \epsilon_{a}\left\langle\mathbf{n}_{k} \cdot \nabla \phi_{k}, \mathbf{w} \cdot \nabla \delta \phi\right\rangle_{0}-2 \epsilon_{0} \epsilon_{a}\left\langle\mathbf{n}_{k} \cdot \nabla \delta \phi, \mathbf{w} \cdot \nabla \phi_{k}\right\rangle_{0}, \\
& \mathcal{L}_{\mathbf{n} \lambda}[\mathbf{w}] \cdot \delta \lambda=2 \int_{\Omega} \delta \lambda\left(\mathbf{n}_{k}, \mathbf{w}\right) d V, \\
& \mathcal{L}_{\phi \mathbf{n}}[\psi] \cdot \delta \mathbf{n}=-2 \epsilon_{0} \epsilon_{a}\left\langle\mathbf{n}_{k} \cdot \nabla \phi k, \delta \mathbf{n} \cdot \nabla \psi\right\rangle_{0}-2 \epsilon_{0} \epsilon_{a}\left\langle\delta \mathbf{n} \cdot \nabla \phi_{k}, \mathbf{n}_{k} \cdot \nabla \psi\right\rangle_{0}, \\
& \mathcal{L}_{\phi \phi}[\psi] \cdot \delta \phi=- 2 \epsilon_{0} \epsilon_{\perp}\langle\nabla \delta \phi, \nabla \psi\rangle_{0}-2 \epsilon_{0} \epsilon_{a}\left\langle\mathbf{n}_{k} \cdot \nabla \delta \phi, \mathbf{n}_{k} \cdot \nabla \psi\right\rangle_{0}, \\
& \mathcal{L}_{\lambda \mathbf{n}}[\gamma] \cdot \delta \mathbf{n}=2 \int_{\Omega} \gamma\left(\mathbf{n}_{k}, \delta \mathbf{n}\right) d V .
\end{aligned}
$$

Additional details are found in [1].

\section{Appendix B. Initial guesses.}

In this appendix, we report the initial guesses used for each example to aid in reproducing the results. Each guess listed here gives the values used on the interior of the domain for all NI levels; the Dirichlet boundary conditions are enforced along the relevant boundaries. In all of the simulations performed, $\lambda$ is initially set to 0 .

In Sections 4.1 and 4.2 , the initial guesses used were $\mathbf{n}=\left(\cos \left(\frac{\pi}{40}\right), \sin \left(\frac{\pi}{40}\right), 0\right)$ and $\mathbf{n}=\left(\cos \left(\frac{\pi}{40}\right),-\sin \left(\frac{\pi}{40}\right), 0\right)$. In addition, the simulations of Section 4.2 use $\phi=V \cdot y$ to initialize the electric potential for both guesses, where $V$ is the potential at the top substrate. 
For Section 4.3, let $\xi_{1}=\left|\tan ^{-1}\left(\frac{0.5-y}{0.5-x}\right)\right|, \zeta_{1}=\frac{9 \pi}{20}$, and define the functions

$$
\begin{aligned}
& n_{1}=\left\{\begin{array}{ll}
\sin \left(\zeta_{1}\right) \cos \left(\xi_{1}\right) & \text { if } x \leq 0.5 \\
-\sin \left(\zeta_{1}\right) \cos \left(\xi_{1}\right) & \text { if } x>0.5,
\end{array} \quad n_{2}= \begin{cases}\sin \left(\zeta_{1}\right) \sin \left(\xi_{1}\right) & \text { if } y \leq 0.5 \\
-\sin \left(\zeta_{1}\right) \sin \left(\xi_{1}\right) & \text { if } y>0.5,\end{cases} \right. \\
& n_{3}=\cos \left(\zeta_{1}\right)
\end{aligned}
$$

Then the two initial values for the director in the section are given by

$$
\mathbf{n}=\left\{\begin{array}{ll}
(0,0,1) & \text { if } x, y=0.5 \\
\left(n_{1}, n_{2}, n_{3}\right) & \text { otherwise, }
\end{array} \quad \mathbf{n}= \begin{cases}(0,0,1) & \text { if } x, y=0.5 \\
\left(n_{1}, n_{2},-n_{3}\right) & \text { otherwise }\end{cases}\right.
$$

Finally, for Section 5.2 , let $\xi_{2}=\frac{7 \pi}{16}$ and $\zeta_{2}=\frac{\pi}{4}$. The initial values for $\mathbf{n}$ are shown in Table B.1.

\begin{tabular}{|l|l|c|}
\hline \multicolumn{1}{|c|}{ Guess 1} & \multicolumn{1}{|c|}{ Guess 2} & Guess 3 \\
\hline$n_{1}=\cos (\pi / 12)$ & $n_{1}=\sin \left(\xi_{2}\right) \cos \left(\zeta_{2} \cos (4 \pi x)\right)$ & $n_{1}=\cos (2 \pi y) \cos (\pi / 8)$ \\
$n_{2}=\sin (\pi / 12)$ & $n_{2}=\sin \left(\xi_{2}\right) \sin \left(\zeta_{2} \cos (4 \pi x)\right)$ & $n_{2}=\cos (2 \pi y) \sin (\pi / 8)$ \\
$n_{3}=0$ & $n_{3}=\cos \left(\xi_{2}\right)$ & $n_{3}=\sin (2 \pi y)$ \\
\hline
\end{tabular}

Table B.1: Formulas for the initial guesses used in Section 5.2. 